\title{
Spinning flavour branes and fermion pairing instabilities
}

\author{
S. Prem Kumar \\ Department of Physics, \\ Swansea University, \\ Singleton Park, Swansea \\ SA2 8PP, U.K. \\ E-mail: s.p.kumar@swansea.ac.uk
}

\begin{abstract}
We consider probe Dp-branes, $p=3,5,7$, in global $A d S_{5} \times S^{5}$, rotating along an internal direction in the $S^{5}$. These are dual to strongly interacting $\mathcal{N}=4$ SYM on $S^{3}$ with massless fundamental flavours, in the presence of an R-symmetry chemical potential for flavour fermions. For massless, "AdS-filling" Dp-brane embeddings at zero temperature, we find an infinite set of threshold values of the chemical potential at which instabilities are triggered. The onset of instability is always preceded by metastability of the zero density state. From the onset values of the chemical potential, we infer that unstable directions favour a homogeneous condensate of a bilinear made from fermion harmonics, or Cooper pairing. We confirm this picture both numerically and analytically. The linearized analysis showing the appearance of instabilities involves a charged scalar in global AdS space coupled to a (large) constant background gauge potential. The resulting frequency space correlator of the fermion bilinear at strong coupling displays poles in the upper half plane. In contrast, the correlator at zero coupling exhibits Pauli blocking due to occupation of states below the Fermi level, but no instabilities. The end-point of the strong coupling instability is not visible in our setup.
\end{abstract}




\section{Contents}

1. Introduction and summary 2

2. Adding flavours to $\mathcal{N}=4 \mathrm{SYM}$ on $S^{3} \times \mathbb{R}$

2.1 D7-brane probes: Matter content and symmetries 6

2.2 The theory on $S^{3} \times \mathbb{R}$

3. Weak coupling interlude 8

3.1 Weak (zero) coupling with $N_{f} / N$ fixed 9

3.2 Green's function for $\tilde{\psi}_{q} \psi_{q}$ at weak coupling 10

4. Probe D7-branes in Global AdS 12

4.1 Embedding Ansatz 13

4.2 Divergences and renormalization 14

4.3 Solutions 15

4.3.1 Constant Solution 15

4.3 .2 Non-constant solutions 16

4.3.3 Free energy and saddle points 18

5. Analytical results: instabilities 21

5.1 Non-constant massless embeddings 21

5.2 Stability analysis: two-point function for $\tilde{\psi}_{q} \psi_{q}$

6. D5 and D3' flavour branes in global $A d S_{5}$

6.1 D5-brane probes 27

6.1.1 Linearized results: 28

6.1 .2 Stability of $\theta=0$ embedding: 29

6.2 D3' probe branes 29

6.2.1 Two-point function around $\theta=0$ : 31

7. Discussion 32 


\section{Introduction and summary}

The behaviour of strongly coupled systems under extreme densities and temperatures is important in a variety of physical situations. Gauge/gravity dualities [1, 2, 3] present us with an approach towards tackling the physics of such situations, both for pure gauge theories [3, 4] and gauge theories with quark-like matter [5, 6, 0, 8, 9, 10]. Holographic duals of strongly interacting fermionic systems at finite density are of considerable interest in the context of superconducting/superfluid systems [11]. Such dual descriptions can also provide the necessary window into the workings of strongly interacting quark matter. This is particularly relevant for QCD where phase transitions are conjectured to occur precisely at densities where the coupling is strong, rendering perturbative methods ineffective, and where lattice approaches need to surmount the technical obstacle posed by the sign problem.

In this note we explore properties of one such setup involving "probe" Dp-branes $(p=3,5,7)$ in global $A d S_{5} \times S^{5}$, with the branes chosen to spin along a compact internal direction in the $S^{5}$. The dual conformal field theories live on spheres. Imparting a rotation to the Dp-branes in the internal directions allows for a chemical potential (conjugate to a $U(1)$ R-charge) for massless fermion flavour fields transforming in the fundamental representation of the gauge group in the boundary field theory.

One of our original motivations for examining these systems in global AdS spacetime was to make contact with recent studies of the phase diagram of $S U(N)$ gauge theories in finite volume with fermion flavours in the fundamental representation [12, [13]. However, aspects of our setup and the resulting physical picture are possibly also of relevance to "top-down" approaches towards holographic superconductor/superfluid models involving probe branes (e.g. 114, 15, 16, 17, 18, 19]).

The works of [12, 13] found, using a weak coupling analytical approach at large$N$, and small volume lattice simulations of QCD, an exotic phase structure of $S U(N)$ gauge theories as a function of quark/fermion number chemical potential. It was shown that at large- $N$ with a large number of flavours, the systems exhibit an infinite sequence of third-order Gross-Witten transitions, across which, fermion number increases in discrete steps. A jump in fermion number occurs when the chemical potential crosses the energy of an elementary fermion harmonic on the sphere, and the corresponding energy level gets occupied. This jump is accompanied by a spike in the Polyakov loop order parameter and a pair of third-order transitions ${ }^{1}$.

It is natural to ask whether the physics of fermions seen above, can be observed in a setting that has a strong coupling description within a gravity dual framework. The probe brane configurations we consider present us with such a setup. However,

\footnotetext{
${ }^{1}$ Lattice studies of QCD at finite volume [13, showed the same qualitative jumps in quark number correlated with spikes in the Polyakov loop. However, these studies also found certain qualitative differences including the appearance of metastable configurations.
} 
while [12, 13] treated theories with $N_{f}$ and $N$ comparable, we will only explore the theory in the 't Hooft large- $N$ limit with $N_{f} / N \rightarrow 0$.

The main result of our study is that the strongly coupled system in finite volume does not see phenomena associated to the energy levels of individual, elementary fermion states. Instead, the system is unstable to the condensation of composite scalars made from bilinears of fermion harmonics on the sphere. The transitions associated to this occur when the chemical potential times the R-charge approaches twice the energy of a fermion harmonic, eventually triggering an instability towards fermion bilinear condensation. The strong coupling analysis shows that a large enough R-symmetry chemical potential acts as a negative mass squared for the fermion bilinears. As expected, the instabilities persist in the infinite volume limit, and potentially imply a phase with spontaneously broken $U(1)$ R-symmetry in the presence of a chemical potential. A complete description of such a symmetry breaking ground state would provide a holographic model of a superfluid/superconductor. However, within our probe prane ansatz, the instabilities appear to be of a runaway nature and the end-point solution is not visible in the setup. Investigations of spinning flavour branes in the Poincare' patch of $A d S_{5}$ were first carried out in [20, while a different way of inducing R-symmetry breaking in the spinning brane setup, via a probe brane magnetic field, was investigated in [14].

The spinning probe Dp-branes considered here, describe strongly coupled $\mathcal{N}=4$ supersymmetric $S U(N)$ Yang-Mills theory on the three-sphere, coupled to flavour multiplets in a way that preserves eight supersymmetries ( $\mathcal{N}=2$ in four dimensions). When the flavours are massless, the theories possess a global R-symmetry. The angular velocity of the probe D-branes along a compact internal direction acts as a chemical potential for this R-symmetry under which fermions in the flavour multiplets are charged. In the strict limit $N \rightarrow \infty$ and $N_{f}$ fixed, which is the probe limit for the Dp-branes, the R-symmetry is non-anomalous and is a conserved quantum number. Furthermore, since the flavours do not "backreact" on the pure gauge dynamics in this limit, we may consistently choose all the R-charge to be concentrated in the flavour sector alone. The "leaking" of the charge to adjoint matter fields of $\mathcal{N}=4$ SYM is suppressed in the large- $N$ limit. In the gravity picture this means that we can have the probes spinning along the internal directions, whilst the supergravity background is not boosted along the same internal directions.

In addition, in the probe limit, the corresponding field theories remain conformally invariant. The D7-brane probe yields a four dimensional $\mathcal{N}=2$ SUSY gauge theory, whilst the D5- and D3-brane probes give rise to $2+1$ and $1+1$ dimensional "defect" CFTs respectively [21, 22]. In the global AdS description, the defect CFT's are localized on an equatorial $S^{2}$ and $S^{1}$ of the boundary three-sphere, and the degrees of freedom localized on the defects interact with the $\mathcal{N}=4$ theory on $S^{3}$.

The strong coupling analysis of the probe Dp-brane action reveals that the massless, zero density embedding encounters an infinite sequence of instabilities with 

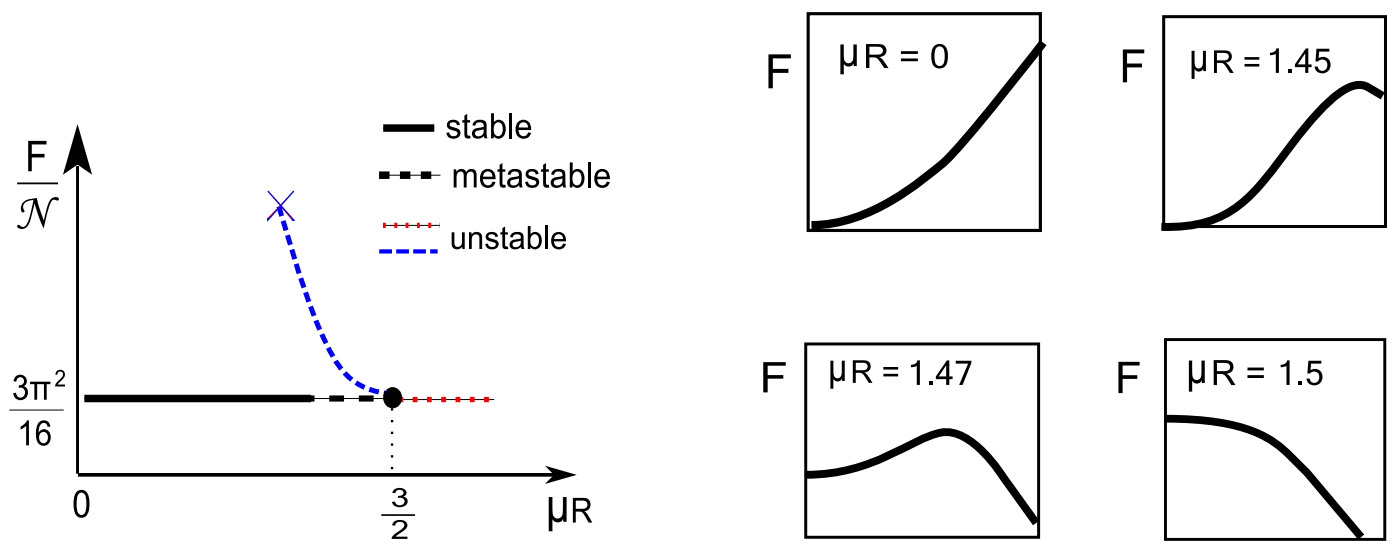

Figure 1: Left: The free energy as a function of chemical potential $\mu$ for the D7 system. The zero density state (solid black) is locally stable as $\mu$ is increased from zero. A new unstable finite density saddle point (dashed blue) with chiral condensate appears when $\mu$ approaches $\frac{3}{2} R^{-1}$. The two merge at $\mu R=\frac{3}{2}$ where an instability develops. $\underline{\text { Right: The }}$ situation is schematically depicted in terms of an effective potential (see also Fig.8).

increasing chemical potential $\mu$, for the $U(1)$ R-symmetry. In particular the instabilities favour the condensation of fermion bilinears with R-charge $Q_{R}=+2$, made up of harmonics of elementary fermions on the sphere. For the D7-branes we find instabilities at

$$
\mu Q_{R}=(2 \ell+1) R^{-1}, \quad \ell=1,2,3, \ldots
$$

while for the defect CFT's on $S^{2}$ and $S^{1}$, the respective onset values are

$$
\mu Q_{R}=2 \ell R^{-1} \quad \text { and } \quad \mu Q_{R}=(2 \ell-1) R^{-1}, \quad \ell=1,2,3, \ldots
$$

The R-charge of the bilinears is $Q_{R}=2$, and in each case the critical value of $\mu Q_{R}$ is twice the energy of an elementary fermion harmonic (on $S^{3}, S^{2}$ and $S^{1}$ ).

The solutions to the non-linear equations of motion of the DBI action, which we analyze numerically, display a somewhat intricate structure in the vicinity of each of these instabilities. The zero density state, which starts off being stable, actually becomes metastable before the onset of the first instability, accompanied by the appearance of a new thermodynamically unstable solution with finite density and fermion bilinear condensate. This latter saddle point eventually merges with the zero density solution at the critical value of the chemical potential. Beyond this value the zero density solution is simply unstable. This phenomenon is summarized in Fig. 1 and in Fig. 8.

Analytical solutions of the linearized equations of motion for the Dp-branes show the emergence of non-trivial embeddings (at linear order) only at the critical values of the chemical potential. This confirms the result of the numerical analysis which 
points towards a merger of two saddle points (a zero density trivial solution and a non-trivial finite density solution) at the critical values of the chemical potential. The linearized equations which lead to non-trivial massless embeddings, are precisely the equations of motion of a charged scalar in global AdS spacetime, coupled to a constant background $U(1)$ gauge potential. For large enough, special values of this background gauge field (the chemical potential), the equations allow for charged fermion bilinear condensates or "scalar hair". In this respect the systems are somewhat similar to holographic superconductor models [11, 23].

The linearized approach also allows the computation of the two-point function for the fermion bilinear which displays an infinite set of discrete poles in the complex frequency plane. At $\mu=0$, the locations of the poles are at twice the energies of the discrete fermion harmonics. With a non-zero chemical potential, the poles migrate and eventually move to the upper half plane, signalling instabilities when onset values of the chemical potential are crossed.

While the detailed features associated to the fermion bilinear instability follow from our anstaz for the probe D-brane action (which only incorporates the so-called "slipping mode" degree of freedom), the end-point of these instabilities does not seem to be captured by this action. This suggests that new effects must be included to obtain a complete description.

The paper is organized as follows. For the purpose of completeness, in Section 2, we review basic field theory features of the probe D7-brane system, paying attention to the introduction of an R-charge chemical potential for flavours alone. In Section 3 , we explain weak coupling features of the theory on a sphere with non-vanishing chemical potential. The probe D7-brane analysis is done in Section 4, with numerical results for the phase diagram and free energies of solutions. Analytical results in the linearized approximation and strong-coupling Green's functions are derived in Section 5. We discuss the rotating D5- and D3'-branes, related to D3/D5 and D3/D3' systems in Section 6, and conclude with speculations on the end-point of the instabilities in Section 7. Various details of weak coupling calculations of the phase structure on $S^{3}$, Green's functions at finite density, and technical features of the D7-brane ansatz are presented in an Appendix.

\section{Adding flavours to $\mathcal{N}=4 \mathrm{SYM}$ on $S^{3} \times \mathbb{R}$}

It is well known that the simplest way of incorporating flavour fields into the AdS/CFT framework is via probe Dp-branes in the bulk type IIB supergravity background dual to, for example, $\mathcal{N}=4 \mathrm{SYM}$ with $S U(N)$ gauge group [5]. The "probe" approximation is valid when the the number of flavour multiplets $N_{f}$ is fixed in the 't Hooft large- $N$ limit.

The presence of flavour fields spoils conformality of the $\mathcal{N}=4$ theory since the gauge coupling now runs and has a putative Landau pole in the ultraviolet (UV). 
In the limit $N_{f} / N \rightarrow 0$ however, the loop contributions from flavour modes are suppressed and the beta function for the 't Hooft coupling, $\lambda=g_{Y M}^{2} N$, is proportional to $\frac{N_{f}}{N}$ and therefore vanishes in the strict large $N$ limit. We may continue to view the theory as being conformal, provided the hypermultiplets have vanishing bare masses. It is natural for CFTs in four dimensions to be viewed as field theories formulated on a spatial three-sphere, a picture that follows from radial quantization on $\mathbb{R}^{4}$. The radius of the three-sphere provides a new scale, allowing for the possibility of non-trivial thermodynamics as a function of temperature and/or chemical potentials [24, 25].

There are three distinct cases to be considered, corresponding to probe D7-, D5- and D3-branes. In all cases the resulting system preserves 8 supersymmetries. The D7-brane probes describe the dynamics of $\mathcal{N}=2$ SUSY gauge theory with $N_{f}$ hypermultiplets, while the dynamics of the two other kinds of probe branes yields lower dimensional defect conformal field theories [21, 22]. We will begin with the study of the D7-brane probes.

\subsection{D7-brane probes: Matter content and symmetries}

The matter content and the interactions of the field theory are most easily summarized in the language of $\mathcal{N}=1$ superfields on $\mathbb{R}^{3,1}$. Each $\mathcal{N}=2$ hypermultiplet consists of two $\mathcal{N}=1$ chiral superfields $(Q, \tilde{Q})$ transforming in the $(N, \bar{N})$ representation of the $S U(N)$ gauge group. The superfields in the hypermultiplet, each consist of a squark and a quark $Q \equiv\left(q, \psi_{q}\right)$ and $\tilde{Q} \equiv\left(\tilde{q}, \tilde{\psi}_{q}\right)$. The quarks $\psi_{q}$ and $\tilde{\psi}_{q}^{\dagger}$ are left- and right-handed Weyl fermions respectively.

With $N_{f}$ hypermultiplets $\left(Q^{i}, \tilde{Q}_{i}\right)$ and using the $\mathcal{N}=1$ SUSY notation, the superpotential of the theory with flavours on $\mathbb{R}^{3,1}$, can be written as

$$
W=\frac{1}{g_{Y M}^{2}}\left(\sum_{i=1}^{N_{f}}\left(\sqrt{2} \tilde{Q}_{i} \Phi_{3} Q^{i}+m \tilde{Q}_{i} Q^{i}\right)+\sqrt{2} \operatorname{Tr}\left(\Phi_{3}\left[\Phi_{1}, \Phi_{2}\right]\right)\right) .
$$

The six adjoint scalars of the $\mathcal{N}=4$ theory, and their superpartners are packaged into the three (complex) chiral multiplets $\Phi_{1}, \Phi_{2}$ and $\Phi_{3}$. Upon coupling to the $\mathcal{N}=2$ hypermultiplets as in the above superpotential, $\Phi_{3}$ naturally gets identified with the scalar component of the $\mathcal{N}=2$ vector multiplet, while $\Phi_{1,2}$ together make up an $\mathcal{N}=2$ adjoint hypermultiplet.

Although one may allow for bare masses for the hypermultiplets, we will only be interested in the theory with massless quarks. In this case the theory has the following global symmetry group

$$
G_{F} \simeq U(1)_{B} \times S U\left(N_{f}\right) \times S U(2)_{R} \times U(1)_{R} \times S U(2)_{\Phi} .
$$

The multiplets $Q^{i}$ transform in the fundamental representation of the flavor symmetry $U\left(N_{f}\right) \simeq U(1)_{B} \times S U\left(N_{f}\right)$, while $\tilde{Q}_{i}$ transform in the conjugate representation. 
The $S U(2)_{R} \times U(1)_{R}$ factor is the R-symmetry group of the $\mathcal{N}=2$ gauge theory. The $S U(2)_{R}$ subgroup acts on the doublets $\left(q^{i}, \tilde{q}^{\dagger i}\right)$ and leaves all other fields invariant. We will be interested in the $U(1)$ subgroup of the R-symmetry. Under a $U(1)_{R}$ transformation, the scalar component of $\Phi_{3}$ has charge +2 , whilst the squarks in the hypermultiplets are uncharged. Importantly, the fermions $\psi_{q}^{i}$ and $\tilde{\psi}_{q i}^{\dagger}$ each carry R-charges +1 and -1 respectively. The $U(1)_{R}$ rotation is therefore an axial symmetry acting on the fermions ${ }^{2}$.

The $U(1)_{R}$ symmetry is anomalous for a fixed number of colours and flavours. However, $\mathcal{N}=2$ supersymmetry relates this anomaly to the beta function for the gauge theory. Like the beta function, the $U(1)_{R}$ anomaly is also parametrically suppressed in the large $N$ limit with $N_{f}$ fixed. It is thus consistent to treat the $U(1)_{R}$ as a symmetry of the theory in the 'tHooft large- $N$ limit.

\subsection{The theory on $S^{3} \times \mathbb{R}$}

When the $\mathcal{N}=2$ SUSY gauge theory described above is formulated on a spatial three-sphere, conformal invariance requires that we add to the Lagrangian of the theory, a coupling of the scalar fields to the Ricci scalar of $S^{3}$ :

$$
\mathcal{L} \rightarrow \mathcal{L}+\frac{1}{R^{2}}\left[\sum_{a=1}^{3} \operatorname{Tr}\left|\Phi_{a}\right|^{2}+\sum_{i=1}^{N_{f}}\left(\left|q_{i}\right|^{2}+\left|\tilde{q}_{i}\right|^{2}\right)\right] .
$$

Here $R$ is the radius of the boundary three-sphere. The conserved current associated to the $U(1)_{R}$ symmetry is a sum of two parts: a contribution $J_{A}$ from the charged adjoint matter of the $\mathcal{N}=4$ sector and another piece $J_{F}$ from the R-charged fundamental fermions.

$$
\begin{aligned}
& j_{R}^{\mu}=J_{A}^{\mu}+J_{F}^{\mu} \\
& J_{A}^{\mu}=i \operatorname{Tr}\left(2 \Phi_{3}^{\dagger} \overleftrightarrow{\nabla}^{\mu} \Phi_{3}+\psi^{\dagger} \bar{\sigma}^{\mu} \psi+\lambda^{\dagger} \bar{\sigma}^{\mu} \lambda\right) \\
& J_{F}^{\mu}=-i \sum_{j=1}^{N_{f}}\left(\psi_{q j}^{\dagger} \bar{\sigma}^{\mu} \psi_{q}^{j}-\tilde{\psi}_{q j} \sigma^{\mu} \tilde{\psi}_{q}^{j \dagger}\right) .
\end{aligned}
$$

Chemical potentials for global symmetries can be turned on in the Lagrangian formulation by first imagining that the corresponding global symmetry is gauged and then introducing a background value for the time component of that gauge field. For the $U(1)_{R}$ symmetry above, a chemical potential will shift the Lagrangian as,

$$
\mathcal{L} \rightarrow \mathcal{L}-\mu\left(J_{A}^{0}+J_{F}^{0}\right)-\mu^{2}\left|\Phi_{3}\right|^{2} .
$$

\footnotetext{
${ }^{2}$ We take the fermions $\psi_{q}^{i}$ and $\tilde{\psi}_{q i}^{\dagger}$ to be left and right handed chiral fermions respectively. This is consistent with the Yukawa coupling implied by the superpotential (2.1).
} 
As is well known [26, 27, 28], for $\mu>1 / R$, and in the absence of fundamental flavours, the chemical potential induces a runaway instability in the potential energy for the adjoint scalar $\Phi_{3}$. However, this issue will not be a problem for us as we explain below.

As we will work in the probe limit for flavour branes, the number of flavour hypermultiplets is small, $N_{f} \ll N$, so that the flavours do not "backreact" on the $\mathcal{N}=4$ gauge theory. We then introduce a chemical potential for the $U(1)_{R}$ charge in the flavour sector alone. Strictly speaking, this will not result in an equilibrium configuration since the R-charge carried by the flavour degrees of freedom will eventually leak out to the R-charged adjoint modes of the full theory ${ }^{3}$. However, in the 't Hooft large $N$ limit with $N_{f}$ fixed, this effect is suppressed.
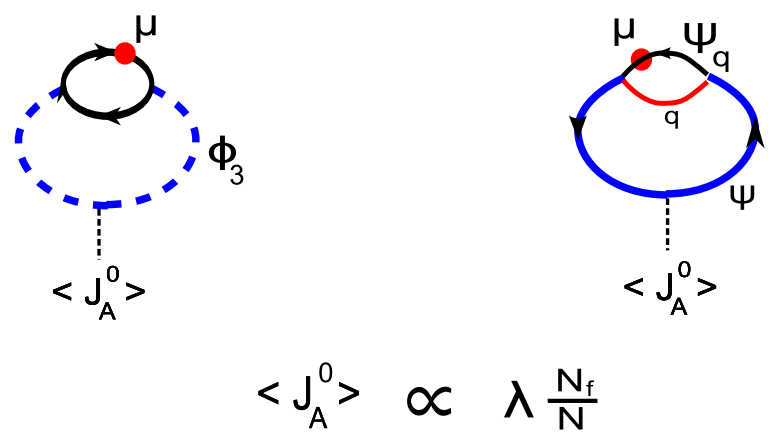

Figure 2: Typical graphs that contribute to a one-point function for $J_{A}^{0}$, the R-charge density in the adjoint sector, when a chemical potential $\mu$ for the flavour R-charge density is introduced. $\Phi_{3}$ and $\psi$ are the complex adjoint scalar and its superpartner, which are both charged under $U(1)_{R}$, while $\left(q, \psi_{q}\right)$ represent flavour squarks and quarks respectively.

As shown in Fig. (2), a chemical potential conjugate to the flavour R-charge density $J_{F}^{0}$ also implies a non-zero one-point function for R-charge density for adjoint matter. These effects are suppressed by flavour loops in the 'tHooft large- $N$ limit. Therefore in this limit, it will be consistent for us to introduce an R-charge chemical potential in the flavour sector alone,

$$
\mathcal{L} \rightarrow \mathcal{L}-\mu J_{F}^{0}
$$

\section{Weak coupling interlude}

For the weakly coupled theory on $S^{3}$, the energies of all the perturbative modes are quantized and there are no zero modes (at zero temperature). The putative zero

\footnotetext{
${ }^{3}$ The spinning D-brane setup has been applied to understand aspects of this non-equilibrium system with massive hypermultiplets in flat space 29. In this case the rotation of the branes gives rise to a time-dependent phase for the mass.
} 
modes for scalars are lifted by the conformal coupling of the scalars to the curvature of the sphere. The eigenfunctions of the spinor Laplacion on $S^{3}$ have the eigenvalues and degeneracies (see e.g. [30])

$$
\varepsilon_{\ell}=\left(\ell+\frac{1}{2}\right) R^{-1}, \quad d_{\ell}=2 \ell(\ell+1), \quad \ell=1,2, \ldots
$$

At large- $N$, for a non-anomalous R-symmetry, the chemical potential for the $U(1)_{R}$ symmetry in the flavour sector is conjugate to the chiral fermion number, which is the number of left-handed Weyl fermions $N_{L}$ minus the number of right-handed fermions $N_{R}$. At zero temperature, as $\mu$ is increased past each of the energy levels (3.1), the quantity $N_{L}-N_{R}$ will undergo a discrete jump,

$$
\Delta\left(N_{L}-N_{R}\right)=d_{\ell} N_{f} N
$$

as the energy levels below $\mu$ get filled.
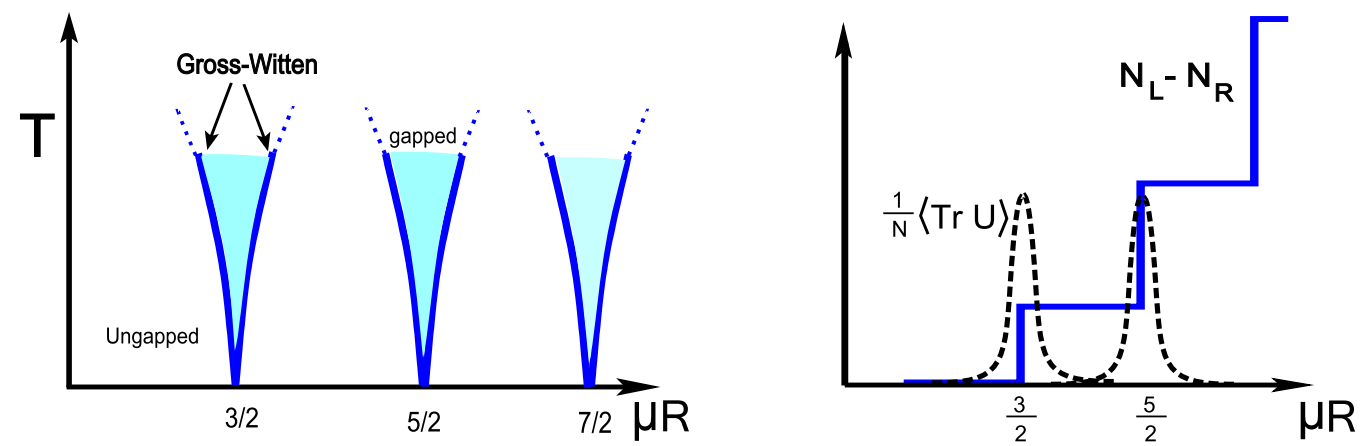

Figure 3: Left: The low $T$, phase structure at zero coupling and large $N$ with fixed $N_{f} / N$ on $S^{3}$. Right: Behaviour of $N_{L}-N_{R}$ and the Polyakov loop with increasing $\mu$ at low $T$.

\subsection{Weak (zero) coupling with $N_{f} / N$ fixed}

The effect is particularly intricate in the Veneziano limit (assuming for the present purposes that the axial $U(1)$ symmetry is anomaly free) on the three-sphere. At nonzero temperatures, the Euclidean theory on $S^{3} \times S^{1}$ reduces to a unitary matrix model for the Polyakov loop matrix $U \equiv \exp i \oint_{S^{1}} A_{0}$. This effective theory is obtained by integrating out all massive Kaluza-Klein modes on $S^{3}$ (e.g [25]). As is well known [25] from this analysis, the free theory without fundamental matter experiences a Hagedorn/deconfinement transition at large- $N$. In particular, at low temperatures the theory is in a confined phase with $\frac{1}{N}\langle\operatorname{Tr} U\rangle=0$, while beyond the deconfinement transition this order parameter acquires a non-zero expectation value.

Incorporating a large number $N_{f}$ of fundamental matter fields with $N_{f} / N$ fixed, changes the low temperature picture, especially when a chemical potential for the fermions is introduced. The situations with baryon number chemical potential [12] 
and $U(1)_{R}$ chemical potential (see Appendix A) for the fermions are quite similar, although the respective technical details differ.

From the analysis in Appendix A, we see that as the flavour R-charge chemical potential $\mu$ is increased towards the energy level $\varepsilon_{\ell}$ of a fermion mode, the theory experiences a third-order Gross-Witten transition [31] at

$$
\mu_{-}^{*} \simeq \varepsilon_{\ell}-T \ln \left(\frac{N_{f}}{N} d_{\ell}\right)
$$

Here, the low temperature approximation implies that $\varepsilon_{\ell} \gg T$. At this transition, the large- $N$ distribution of the eigenvalues of the unitary matrix $U$ changes from an ungapped to a gapped phase. The distribution, however, quickly reverts to its ungapped phase as $\mu$ is increased slightly past $\varepsilon_{\ell}$, at

$$
\mu_{+}^{*} \simeq \varepsilon_{\ell}+T \ln \left(\frac{N_{f}}{N} d_{\ell}\right)
$$

which represents yet another Gross-Witten transition. This pair of transitions is accompanied by two qualitatively interesting effects. The first is a change in the Polyakov loop order parameter $\frac{1}{N}\langle\operatorname{Tr} U\rangle$ which is small in the ungapped phases, but becomes large in the gapped phase. The second physical effect is the chiral fermion number which jumps across the two transitions so that $\Delta\left(N_{L}-N_{R}\right)=d_{\ell} N N_{f}$. These phenomena are depicted in Figure (3). The work of [12 has shown that these qualitative effects (with a baryon number chemical potential) persist in QCD $(N=3)$ in a small volume.

\subsection{Green's function for $\tilde{\psi}_{q} \psi_{q}$ at weak coupling}

The study of the large- $N$ theory at strong coupling and with $N_{f} / N$ fixed is beyond the scope of this paper. The main point of the weak coupling picture described above is to indicate the various effects associated to threshold values of the chemical potential. As we will see below, the elementary fermionic states are not visible at strong coupling; instead, the fluctuations of probe D-branes at strong coupling comprise of composite gauge invariant operators made up of fundamental fermions and their superpartners. The simplest amongst these is the fermion bilinear

$$
\mathcal{O} \equiv \sum_{i=1}^{N_{f}} \tilde{\psi}_{q i} \psi_{q}^{i} .
$$

This is a dimension three operator at weak coupling. In the context of the D3D7 system its conformal dimension is protected by its R-charge [5]. However, the normalization of its correlator can and does receive quantum corrections as a function of $\lambda=g_{Y M}^{2} N$. At strong coupling, in the presence of an R-charge chemical potential, we will be interested in computing the two-point function of this operator on $S^{3}$. It will be extremely interesting to contrast this with perturbative results. The frequency 


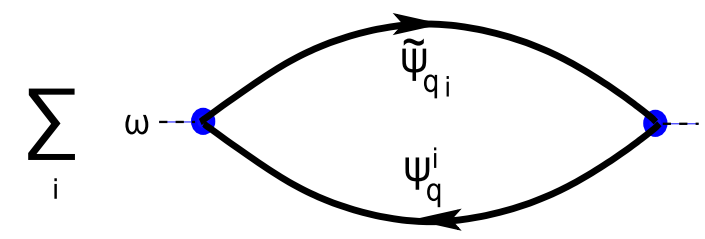

Figure 4: Two-point correlator of $\tilde{\psi}_{q} \psi_{q}$ in the free theory, in frequency space.

space retarded correlator of $\mathcal{O}$, at zero external spatial momentum is,

$$
\tilde{\mathcal{G}}_{R}(\omega)=i \int_{0}^{\infty} d t e^{i \omega t} \int \frac{d^{3} x}{\operatorname{Vol}}\langle[\mathcal{O}(\vec{x}, t), \mathcal{O}(0)]\rangle
$$

Using the finite density propagators (B.5) for the internal fermions in Fig.(4), we obtain a general form for this correlator at zero temperature and non-zero chemical potential $\mu>0$

$$
\tilde{\mathcal{G}}_{R}(\omega)=\frac{N_{f} N}{2 \pi^{2} R^{3}} \sum_{\ell=1}^{\infty} d_{\ell}\left(\frac{1}{\omega+2 \varepsilon_{\ell}}-\frac{1}{\omega-2 \varepsilon_{\ell}}\right)\left[1-2 \Theta\left(\mu-\varepsilon_{\ell}\right)\right] .
$$

For the retarded correlator we need to choose an " $i \epsilon$ " prescription where the poles in the correlator are taken to be slightly below the real axis. The simple poles in $\omega$ represent on-shell intermediate modes with vanishing external spatial momentum. The step function $\Theta\left(\mu-\varepsilon_{\ell}\right)$ is the Fermi-Dirac distribution evaluated at zero temperature, and exhibits the "Pauli-blocking" of occupied levels below the energy $\mu$. Using the energy levels $\varepsilon_{\ell}$ and degeneracies for $S^{3}$, the Green's function can be written in terms of digamma functions (up to constants and "contact" terms proportional to $\left.\omega^{2}\right)$

$$
\begin{aligned}
& \tilde{\mathcal{G}}_{R}(\omega)=\frac{N_{f} N}{16 \pi^{2} R^{2}}\left(\omega^{2} R^{2}-1\right) {\left[\psi\left(\frac{1}{2}-\frac{1}{2} \omega R\right)+\psi\left(\frac{1}{2}+\frac{1}{2} \omega R\right)\right.} \\
& n \equiv\left[\mu R-\frac{1}{2}\right] .\left.-2 \psi\left(\frac{3}{2}+n+\frac{1}{2} \omega R\right)-2 \psi\left(\frac{3}{2}+n-\frac{1}{2} \omega R\right)\right] \\
& n \equiv
\end{aligned}
$$

Here $n$ is the largest integer less than or equal to $\mu R-\frac{1}{2}$. In the perturbative limit, the chemical potential does not introduce new poles in the correlator $\tilde{\mathcal{G}}(\omega)$. In particular, as expected there are no instabilities of any kind. In the limit of large $R$, we should recover the correlator in flat space, after incorporating the correct $i \epsilon$ prescription,

$$
\tilde{\mathcal{G}}_{R}(\omega) \rightarrow-\frac{N_{f} N}{16 \pi^{2}} \omega^{2}\left[\ln \left(\omega^{2} R^{2}\right)+\ln \left(\frac{4 \mu^{2}-\omega^{2}}{\omega^{2}}\right)^{2}+2 i \pi \operatorname{sgn}(\omega) \Theta\left(\omega^{2}-4 \mu^{2}\right)\right]
$$


using $\psi(x) \rightarrow \ln x$ for large $x$. The correlator has branch points at $\omega= \pm 2 \mu$ and an imaginary part for $\omega^{2}>4 \mu^{2}$. This is because states with energy below $|\mu|$ are occupied and therefore Pauli-blocked. Only when the external energy $|\omega|$ is larger than $2|\mu|$, can the intermediate states be on-shell and yield an imaginary part for the Green's function. When $\mu=0$, the correlator has the expected behaviour $\sim \omega^{2} \ln \omega$ for a dimension three operator. In particular, at $\mu=0$ we expect the two-point function (including when $R$ is finite) to have the same form for all values of the 't Hooft coupling, although the normalization can and does receive quantum corrections. We now turn to the strong coupling description of the system in the probe limit $N_{f} \ll N$.

\section{Probe D7-branes in Global AdS}

In the large- $N$ limit and at strong 't Hooft coupling $\lambda=g_{Y M}^{2} N \gg 1$, the flavour modes correspond to low-energy excitations of probe D7-branes in the $A d S_{5} \times S^{5}$ geometry [5]. The flavour D7-branes wrap an $S^{3} \subset S^{5}$ while filling the $A d S_{5}$ directions. Such embeddings of the D7-brane are characterized by the "slipping angle" $\theta$, which determines the size of the three-sphere wrapped by the D7-brane and is defined by the following paramerization of the $S^{5}$ metric

$$
d \Omega_{5}^{2}=d \theta^{2}+\sin ^{2} \theta d \phi^{2}+\cos ^{2} \theta d \Omega_{3}^{2}, \quad 0 \leq \theta \leq \frac{\pi}{2} .
$$

A static probe D7-brane wrapping this $S^{3}$ breaks the $S O(6)$ R-symmetry of $\mathcal{N}=4$ theory to an $S U(2)_{R} \times U(1)_{R}$ subgroup. As discussed above, the $U(1)_{R}$ is non-

anomalous in the theory with $\frac{N_{f}}{N} \rightarrow 0$ and is the isometry generated by shifts of the angle $\phi$. We will consider probe branes spinning in the $U(1)_{R}$ isometry direction parametrized by the angle $\phi$. The associated angular velocity is conjugate to the $U(1)_{R}$ charge carried by such states.

When embedded in the global AdS spacetime, the D7-brane world-volume is topologically $S^{3} \times S^{3} \times \mathbb{R} \times \mathbb{R}_{t}$. The first $S^{3}$ sits inside the global $A d S_{5}$ geometry whose conformal boundary is an $S^{3}$. The D7-brane wraps a second $S^{3} \subset S^{5}$, specified by the polar angle $\theta$ in Eq. (4.1). We write the global $A d S_{5}$ metric in FeffermanGraham coordinates 32

$$
d s^{2}=-\frac{\left(1+\frac{1}{4} z^{2}\right)^{2}}{z^{2}} d t^{2}+\frac{\left(1-\frac{1}{4} z^{2}\right)^{2}}{z^{2}} d \Omega_{3}^{2}+\frac{d z^{2}}{z^{2}}
$$

with $0<z \leq 2$. The conformal boundary is approached as $z \rightarrow 0$. We have set the AdS radius to unity. In these units and the coordinate system employed above, the radius of the boundary three-sphere is $R=1$. 


\subsection{Embedding Ansatz}

We can take the D7-brane to spin with constant angular velocity in the $\phi$ direction, while at the same time allowing for a radial dependence of its angular coordinate on the $S^{5}$ [14, 20, 33,

$$
\phi=\tilde{\mu} t+g(z) .
$$

It turns out (Appendix C) that for massless flavours the radial dependence must be taken to vanish. So we will only consider the configurations with $g(z)=0$, as we are mainly interested in the $m=0$ theory when the $U(1)_{R}$ can be viewed as a symmetry (at least in the large- $N$ limit). The angular velocity $\tilde{\mu}$ is related to the R-charge chemical potential in field theory, as

$$
\tilde{\mu}=2 \mu \text {. }
$$

The factor of two appears because the two fields excited on the probe brane, namely $\theta$ and $\phi$ are related to the magnitude and phase, respectively, of the fermion bilinear made from flavour fields. This object has R-charge +2 . An R-charge chemical potential for a fundamental fermion flavour can also be viewed as a time dependent phase $\exp (i \mu t)$ for the fermion fields. Correspondingly, the bilinears have a time dependent phase $\exp (2 i \mu t)$.

Setting $g(z)=0$ for massless flavours, the induced metric on the D7-brane is

$$
\begin{aligned}
& \left.d s^{2}\right|_{\mathrm{D} 7}= \\
& -\left(\frac{\left(1+\frac{1}{4} z^{2}\right)^{2}}{z^{2}}-4 \mu^{2} \sin ^{2} \theta\right) d t^{2}+\left(z^{2} \theta^{\prime}(z)^{2}+1\right) \frac{d z^{2}}{z^{2}}+\frac{\left(1-\frac{1}{4} z^{2}\right)^{2}}{z^{2}} d \Omega_{3}^{2}+\cos ^{2} \theta d \tilde{\Omega}_{3}^{2} .
\end{aligned}
$$

The D7-brane probe dynamics is then controlled by the DBI action

$S_{\mathrm{D} 7}=2 \pi^{2} \mathcal{N}_{\mathrm{D} 7} \int d t d z \cos ^{3} \theta(z) \frac{\left(1-\frac{1}{4} z^{2}\right)^{3}}{z^{5}} \sqrt{\left(1+z^{2} \theta^{\prime}(z)^{2}\right)\left(\left(1+\frac{1}{4} z^{2}\right)^{2}-4 \mu^{2} z^{2} \sin ^{2} \theta\right)}$,

where $\mathcal{N}_{\mathrm{D} 7}$ depends on the D-brane tension $T_{\mathrm{D} 7}$ and the number of flavours $N_{f}$, as

$$
\mathcal{N}_{D 7} \equiv 2 \pi^{2} N_{f} T_{\mathrm{D} 7}=\frac{\lambda}{2(2 \pi)^{4}} N_{f} N
$$

The additional normalization factor of $2 \pi^{2}$ in Eq. (4.6), can be traced to the volume of the boundary three-sphere, whose radius is 1 in our units.

Classical solutions to the equations of motion resulting from varying Eq. 4.6) have the following general behaviour near the AdS boundary,

$$
\left.\theta(z)\right|_{z \rightarrow 0}=\theta_{(0)} z+\theta_{(2)} z^{3}+\frac{1}{2} \theta_{(0)}\left(1-4 \mu^{2}\right) z^{3} \ln z+\ldots
$$

From the standard AdS/CFT dictionary [2] this is the behaviour expected for a supergravity mode dual to a dimension 3 operator in the field theory. It is interesting 
to note that in global AdS, there is an extra logarithmic term in the asymptotic expansion which explicitly depends on the chemical potential. This will give rise to a chemical potential dependent counterterm for the probe brane action below. The operator must be made from flavour fields and must transform under the global $U(1)_{R}$ symmetry. It is natural then, to associate the slipping angle $\theta$ with the quark bilinear $^{4}$

$$
\mathcal{O}=\sum_{i=1}^{N_{f}} \tilde{\psi}_{q i} \psi_{q}^{i}
$$

The leading coefficient $\theta_{(0)}$, when non-zero, results in a bare quark (hypermultiplet) mass which explicitly breaks the $U(1)_{R}$ symmetry. For vanishing hypermultiplet mass, the coefficient of the $z^{3}$ term directly yields the quark condensate $\langle\mathcal{O}\rangle$. A nonzero quark condensate breaks the $U(1)_{R}$ symmetry spontaneously (assuming that the quark mass is zero). The precise dictionary between the gauge theory parameters and supergravity modes is quoted below.

\subsection{Divergences and renormalization}

The D7-brane probe action is formally divergent and needs to be regulated and renormalized. The regularization of these divergences is well understood within the general framework of the holographic-RG approach applied to probe D-branes [34, 35, 36. It is instructive to first look at all the divergent counterterms implied by the near-boundary asymptotics (4.13) in the D7-brane action, and then compare with the results of the holographic RG method. By cutting off the $z$ integration at $z=\epsilon \ll 1$, we find the following divergent terms

$$
S_{\mathrm{D} 7}^{\epsilon} \simeq 2 \pi^{2} \mathcal{N}_{\mathrm{D} 7} \int d t\left(-\frac{1}{4 \epsilon^{4}}+\frac{1}{4 \epsilon^{2}}+\frac{\theta_{(0)}^{2}}{2 \epsilon^{2}}+\theta_{(0)}^{2}\left(1-4 \mu^{2}\right) \ln \epsilon+\ldots\right)
$$

Note that the chemical potential $\mu$ contributes to the logarithmically divergent counterterm. However, when $\theta_{(0)}=0$ and the hypermultiplets are massless, the divergences are $\mu$-independent. This is consistent with the general expectation that much like a finite temperature, a chemical potential should not introduce new divergences in the theory. For non-zero hypermultiplet masses, $U(1)_{R}$ is not a good symmetry and the angular velocity $\mu$ leads to a time-dependent mass and also makes an appearance in the UV divergent terms.

The divergences are removed by the addition of local counterterms at $z=\epsilon$ to yield the regularized action

$$
S^{\mathrm{reg}}=S_{\mathrm{D} 7}^{\epsilon}+\mathcal{N}_{\mathrm{D} 7} \int d \Omega_{3} d t \sum_{k=1}^{4} L_{k}
$$

\footnotetext{
${ }^{4}$ The precise mapping relates $\theta$ to the operator $\tilde{\psi}_{q i} \psi_{q}^{i}+q_{i}^{\dagger} \Phi_{3} q^{i}+\tilde{q}_{i} \Phi_{3} \tilde{q}^{\dagger}$.
} 
Denoting the boundary metric of the cut off AdS space as $\gamma_{\mu \nu} d x^{\mu} d x^{\nu} \equiv \frac{1}{\epsilon^{2}}\left(-d t^{2}+\right.$ $d \Omega_{3}^{2}$ ), we can write these counterterms in terms of the Ricci scalar $\mathcal{R}$ of the boundary at $z=\epsilon$ as in [34, 35, 36]:

$$
\begin{array}{ll}
L_{1}=-\frac{1}{4} \sqrt{-\gamma}, \quad L_{2}=\frac{1}{24} \sqrt{-\gamma} \mathcal{R}, & L_{3}=0, \quad L_{4}=\frac{1}{2} \sqrt{-\gamma} \theta(\epsilon)^{2} \\
L_{5}=\sqrt{-\gamma}\left(\frac{1}{6} \mathcal{R}+4 \mu^{2} \gamma^{t t}\right) \theta(\epsilon)^{2} \ln \theta(\epsilon), & L_{6}=-\frac{5}{12} \sqrt{-\gamma} \theta(\epsilon)^{4} .
\end{array}
$$

We have used the fact that $\theta(\epsilon)$ is given by Eq.(4.13) as $\epsilon \rightarrow 0$ and that $\mathcal{R}=6 \epsilon^{2}$. Notice that the counterterm $L_{5}$ depends on the chemical potential $\mu$. The form of this counterterm can be deduced by first noting that that $\theta$ and $\phi$ can be packaged into a complex scalar $\Phi$ and the counterterms for this scalar can be expressed in the form of Eq.(4.2) in [34] ${ }^{5}$.

Having defined the regulated action, the dictionary between the gauge theory parameters, namely the quark mass and condensate, and corresponding supergravity modes can be worked out easily (having set the AdS radius to unity). Specifically, for massless hypermultiplets we obtain

$$
m=\frac{\theta_{(0)}}{2 \pi \alpha^{\prime}}=0, \quad\left\langle\tilde{\psi}_{q} \psi_{q}\right\rangle=\mathcal{N}_{\mathrm{D} 7} \theta_{(2)}
$$

\subsection{Solutions}

The DBI equations of motion have a large family of spinning solutions. The solutions fall into two topologically distinct varieties. In one class of solutions, the $S^{3} \subset S^{5}$ which is wrapped by the D7-brane probe, shrinks in the interior, before the brane reaches the centre of the global AdS space. This type of solution always yields a non-zero hypermultiplet mass which can be read off from the asymptotic behaviour of $\theta(z)$. The second category of solutions are those where the second $S^{3}$ embedded in $A d S_{5}$ shrinks, and the probe brane reaches the origin of AdS. A very interesting topology-changing, but continuous phase transition between these two classes of solutions was discovered in 36, 37. This phase transition was originally found and explained at zero temperature, at a critical value of the hypermultiplet mass. It can be thought of as a finite volume, meson binding/unbinding transition at strong coupling and zero temperature. The second category of solutions - the AdS-filling embeddings, include configurations corresponding to massless hypermultiplets. In this phase, a maximally separated static quark-antiquark pair is stable against light quark pair production. We will be interested primarily in the AdS-filling massless embeddings which do not explicitly break the $U(1)_{R}$ symmetry.

\subsubsection{Constant Solution}

The simplest solution to the DBI equations of motion following from Eq. (4.6) is $\theta(z)=0$. This has $m=\langle\mathcal{O}\rangle=0$. The free energy or the action per unit time for the

\footnotetext{
${ }^{5}$ I would like to thank Andy O' Bannon for pointing this out to me.
} 
constant solution is

$$
F=2 \pi^{2} \mathcal{N}_{\mathrm{D} 7}\left[\int_{\epsilon}^{2} d z \frac{1}{z^{5}}\left(1-\frac{1}{4} z^{2}\right)^{3}\left(1+\frac{1}{4} z^{2}\right)-\frac{1}{4 \epsilon^{4}}+\frac{1}{4 \epsilon^{2}}\right]=\frac{3 \pi^{2}}{16} \mathcal{N}_{\mathrm{D} 7} .
$$

The induced metric on the D7-brane is simply (global) $A d S_{5} \times S^{3}$. The charge density conjugate to $\mu$

$$
d \equiv \mathcal{N}_{\mathrm{D} 7} \int_{0}^{2} d z \frac{\partial \mathcal{L}}{\partial \mu}
$$

evaluates to zero for the $\theta=0$ solution.

The trivial solution with $m=0$ and zero R-charge density $(\theta(z)=0)$ exists for all values of $\mu$, but as we will see below, it is not always thermodynamically stable.

\subsubsection{Non-constant solutions}

We will now examine in detail the possibility that there may be additional, nonconstant solutions to the DBI equations of motion with $m=\theta_{(0)}=0$. If such embeddings were to exist, they would correspond to configurations with a finite Rcharge density and which also spontaneously break the $U(1)$ R-symmetry by the formation of a fermion bilinear condensate $\theta_{(2)} \neq 0$.

To find out whether such configurations exist, given the non-linearity of the DBI action and equations of motion, we first employ a brute force numerical approach. For non-constant solutions which fill AdS space ${ }^{6}$, smoothness at the origin of $A d S_{5}$ requires

$$
\left.\theta(z)\right|_{z=2}=\Upsilon=\text { constant } ;\left.\quad \theta^{\prime}(z)\right|_{z=2}=0
$$

Furthermore, reality of the DBI action (4.6) imposes the restriction

$$
\left.\sin \theta(z)\right|_{z=2} \leq \frac{1}{2 \mu}
$$

so that the action is real. At this point we stress that we only focus attention on the solutions having $\phi=2 \mu t+g(z)$ with $g(z)=0$. This is because we want to isolate the $m=0$ embeddings and for these $g(z)$ must necessarily vanish, as argued in Appendix C. For large enough $\mu$, we may expect $\theta(z)$ to be small everywhere, and apply a linearized approximation to the equation of motion. We will return to this shortly.

The full space of non-trivial spinning D7-brane embeddings for general $\mu$, includes both the AdS-filling solutions and the solutions which cap off before getting to the orgin of $A d S_{5}$. The latter class of embeddings are characterized by the fact that the $S^{3} \subset S^{5}$ wrapped by them shrinks smoothly before the brane gets to the centre of $A d S_{5}$ :

$$
\theta\left(z_{0}\right)=\frac{\pi}{2}, \quad \theta^{\prime}\left(z_{0}\right) \rightarrow \infty, \quad \text { for some } z_{0}<2 .
$$

\footnotetext{
${ }^{6}$ Solutions which cap off before getting to the centre of AdS spacetime, always correspond to massive flavours, and fall in the category of so-called "Minkowski embeddings".
} 
By numerically solving the DBI equations of motion for different values of $\mu$, we extract the mass parameter $m$ and condensate $\left\langle\tilde{\psi}_{q} \psi_{q}\right\rangle$ from the asymptotics (4.13). In this way we can obtain all possible types of D7-brane embeddings which have the slipping angle $\theta(z)$ as the only non-trivial mode. The possible space of solutions is summarized in Fig.(5)

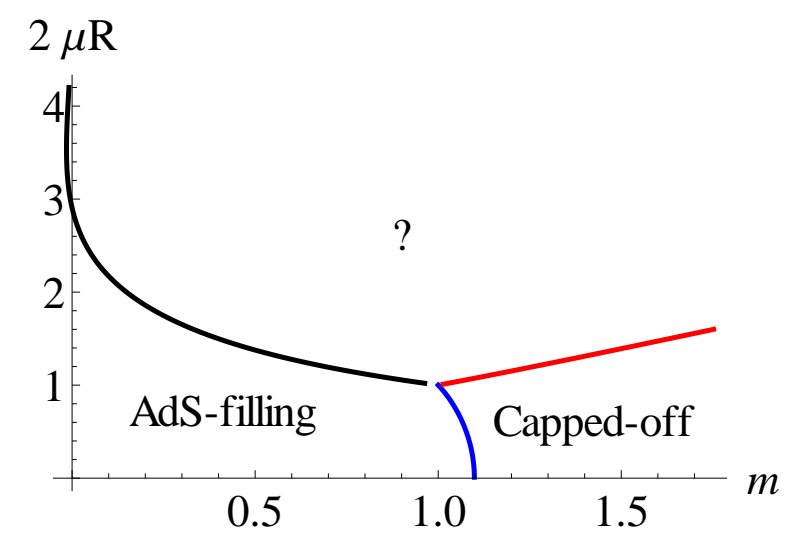

Figure 5: The space of known spinning D7-brane embeddings in global $A d S_{5} \times S^{5}$. The known solutions all lie to one side of the curves shown. The figure also shows that near $\mu R \approx \frac{3}{2}$, there is a solution with $m=0$. (Note that we have reinstated the radius $R$ of the boundary sphere, which was set to 1 by our metric conventions).

The figure exhibits three "phase boundaries":

- The line in blue separates "AdS-filling" solutions from the "capped-off" embeddings. This boundary line is obtained by looking at AdS-filling embeddings with $\left.\theta(z)\right|_{z=2} \rightarrow \pi / 2$ and $\left.\theta^{\prime}(z)\right|_{z=2}=0$. These are on the verge of getting capped off and form a phase boundary for a continuous topology-changing transition of the type found originally in [37, 36].

- The red line is the maximum allowed value of the chemical potential $\mu$, below which the DBI action still remains real, for the massive capped-off solutions or so-called "Minkowski embeddings". This is obtained by noting that a D7-brane ending at $z=z_{0}<2$ with $\theta\left(z_{0}\right)=\pi / 2$ must have $\left(1+\frac{1}{4} z_{0}^{2}\right) \geq 2 \mu z_{0}$ for every allowed value of mass (from (4.6)).

- The third boundary line in black is of interest to us and it represents solutions that fill $A d S_{5}$, with the maximum allowed value of $\mu$ dictated by reality of the DBI action (4.6). These saturate the inequality (4.17), so that $2 \mu=1 /\left.\sin \theta(z)\right|_{z=2}$. A key feature of this family of solutions is that it intersects the $m=0$ axis at several points close to (but not precisely at) odd integer values of $\mu R$, (Fig. (6)). 

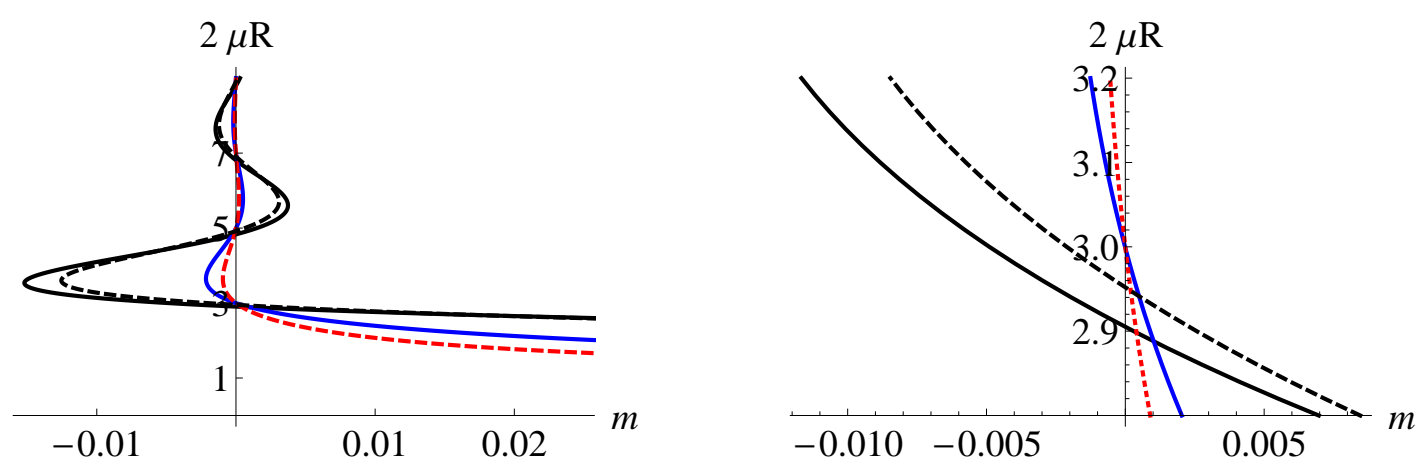

Figure 6: Left: A blown-up plot of the masses and chemical potentials for three families of AdS-filling solutions. The black line is the boundary line depicted in Fig. 4. New massless embeddings appear in the vicinity of $\mu R=\frac{3}{2}, \frac{5}{2}, \frac{7}{2} \ldots$ Right: Closer examination reveals massless $(m=0)$ finite density solutions for a finite range of $2 \mu R$, close to the odd integers.

Blowing up the region for small $m$, we see clearly in Fig. (6), that there exist nontrivial solutions with $m=0$. By plotting the masses for four families of solutions, we see that the non-constant, vanishing mass embeddings occur near $2 \mu R=3,5,7, \ldots$. It is also clear from the plots that there is a finite range of values of $2 \mu R$ in the vicinity of the odd-integer points, for which non-trivial massless solutions arise. For example, it seems that such finite density massless solutions to the DBI equations of motion appear for $2.9 \lesssim 2 \mu R \lesssim 3$.

The four families of solutions, whose masses are displayed in Fig.(6), were obtained by integrating the equations of motion outwards from the origin $(z=2)$ with boundary conditions, assuming that $2 \mu>1$ :

$$
\left.\theta^{\prime}(z)\right|_{z=2}=0, \quad \theta(z=2)=\sin ^{-1}\left(\frac{1}{n(2 \mu-1)+1}\right) \quad n=1,2,15,35 .
$$

The values of $n$ were chosen to illustrate the full range of $\mu$ in Fig.(6), in the vicinity of $2 \mu R=3$, for which new $m=0$ embeddings exist. The limiting case for which the DBI action still remains real, corresponds to $n=1$ (solid black curve). The families labelled by $n=2,15$ and 35, all satisfy Eq.(4.17) and are represented by the dotted-black, solid-blue and dotted-red curves, respectively in Fig.(66).

All the configurations above (including those with $m=0$ ) will necessarily have a non-vanishing chiral condensate $\left\langle\tilde{\psi}_{q} \psi_{q}\right\rangle$, indicating that the solutions with $m=0$, dynamically break the chiral $U(1)_{R}$ symmetry (see Fig.(17)). What remains to be established is whether these configurations are stable and whether they are thermodynamically favoured over the constant $\theta=0$ solution.

\subsubsection{Free energy and saddle points}

We now turn to the key issue, namely, how the free energies of the non-trivial D7brane embeddings discussed above compare with that of the constant $\theta=0$ embed- 

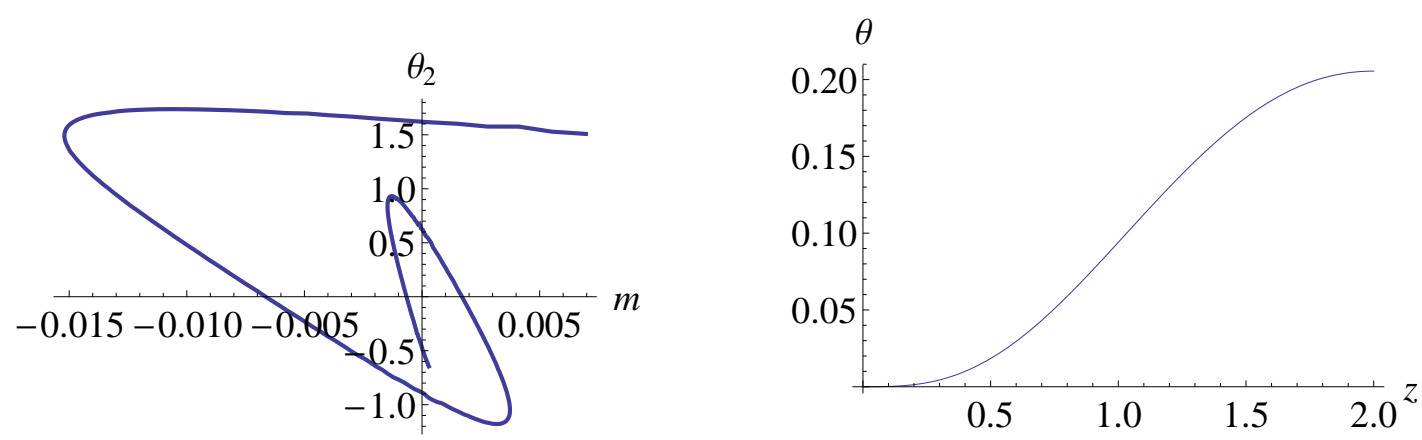

Figure 7: Left: The normalizable mode $\theta_{(2)}$ which is proportional to the chiral condensate $\left\langle\tilde{\psi}_{q} \psi_{q}\right\rangle$ when $m=0$. These are the values extracted from the $n=1$ family of solutions. Right: The non-constant massless solution for $2 \mu R \approx 2.95$.

ding. We first note that, as $\mu R$ is dialed from zero, $\theta=0$ remains the only solution of the DBI equations of motion. A non-constant embedding first appears at $2 \mu R \approx 2.9$ (from the $n=1$ family above).

Using the regulated form of the action (4.11), we numerically evaluate on each of the $m=0$ solutions, the difference of the regulated actions,

$$
\Delta S \equiv\left(2 \pi^{2} \mathcal{N}_{\mathrm{D} 7}\right)^{-1}\left[S^{\mathrm{reg}}(m=0, \mu R)-\left.S^{\mathrm{reg}}\right|_{\theta=0}\right]
$$

In all cases we find that the non-constant embeddings in the window $2.9 \lesssim 2 \mu R \lesssim 3$ have a higher action than the $\theta=0$ solution. Thus these must either be unstable or metastable. In addition, in the limit that $2 \mu R \rightarrow 3$, the non-constant embeddings approach $\theta=0$.

The complete situation is best described by the following procedure leading to Fig.(8). For every non-trivial solution $\theta(z)$, obtained with boundary conditions of the form (4.19), we define a one-parameter family of configurations which do not necessarily solve the DBI equations of motion,

$$
\begin{aligned}
& \left.\theta(z ; s) \equiv s \theta(z)\right|_{m=0, \mu \neq 0} \quad 0 \leq s \leq s_{*}, \\
& \left.s_{*} \theta(z)\right|_{z=2} \equiv \sin ^{-1}\left(\frac{1}{2 \mu}\right) .
\end{aligned}
$$

This is a simple rescaling of a given non-trivial solution, where the rescaling parameter is bounded by the requirement that the DBI action evaluated on the configuration be real. It yields a one dimensional slice of the configuration space of $\theta(z)$ (with the condition that the hypermultiplet mass evaluates to zero at the boundary), along which, only the two points $s=0$ and $s=1$ represent saddle point configurations or solutions of the DBI equations of motion for a fixed $\mu$. It is important that $s$ is bounded by some value $s_{*}$ beyond which the DBI action evaluated on these rescaled 
configurations ceases to be real. Hence the configuration space of $\theta(z)$ has a boundary for some fixed $\mu$.

In Fig. (8) we plot the action evaluated on these configurations and find that the non-constant solutions are always unstable. In contrast, the zero density, $\theta=0$ embedding is stable for low $\mu$, but becomes metastable as $\mu$ is increased, eventually becoming unstable at $2 \mu R \approx 3$. The end-point of the metastability/instability appears to lie outside the region of validity of the ansatz for our probe D-brane setup. The main result we take away from this analysis is that the $\theta=0$ solution becomes
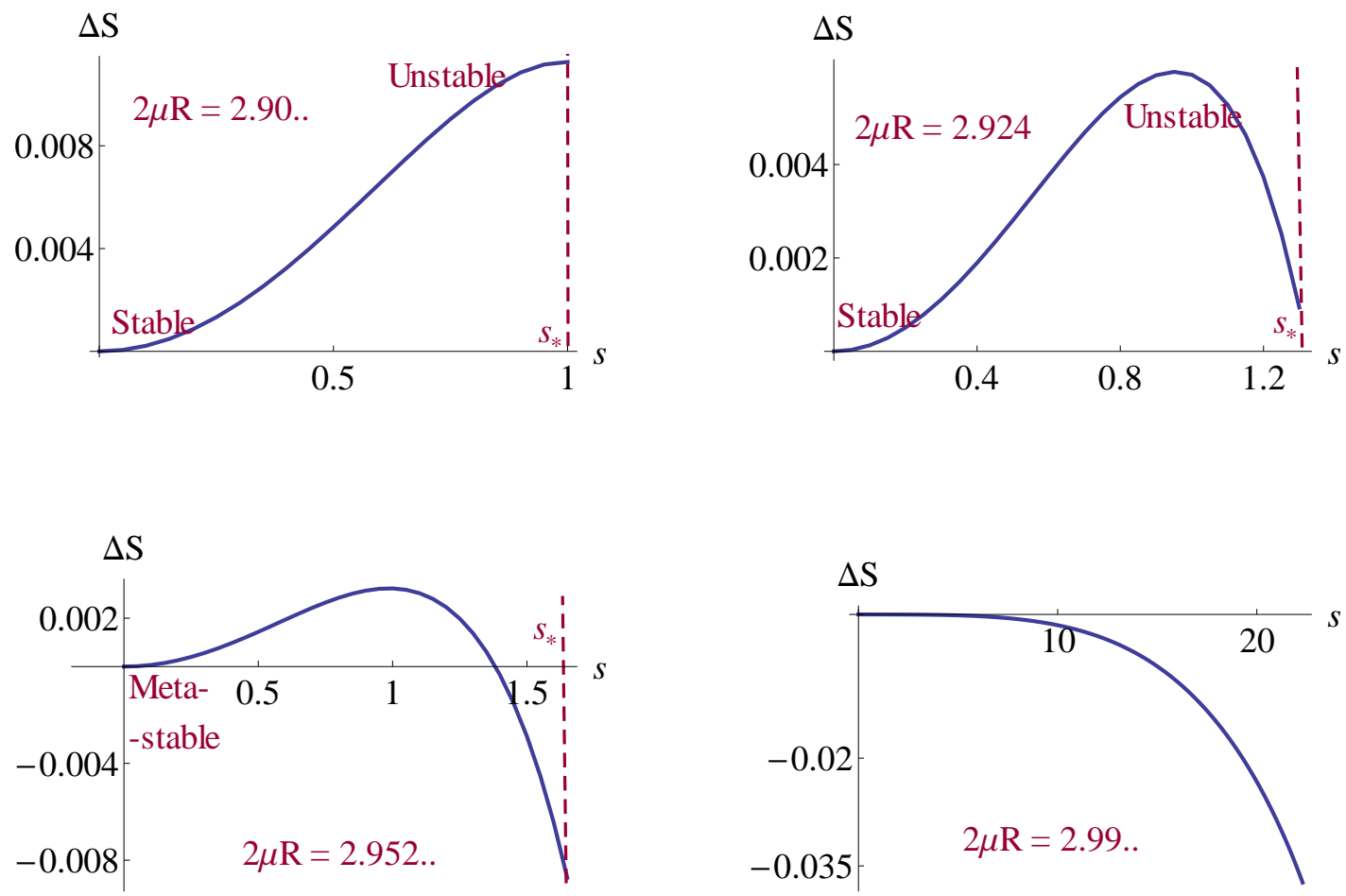

Figure 8: $\Delta F$, the difference in free energy (action per unit time) between non-constant massless configurations and the zero density state, evaluated along a one-parameter family of configurations labelled by $s$. The point $s=0$ represents $\theta(z)=0$, while $s=1$ corresponds to the new non-constant solution which first appears when $2 \mu R \gtrsim 2.9$. The four plots illustrate what happens when $\mu R$ is increased. At $2 \mu R=3$, the critical point, the two saddles merge and the system has a runaway instability.

metastable and eventually encounters a runaway instability at $2 \mu R=3$.

The entire picture described in this section is repeated for every odd-integer value of $2 \mu R$. The unstable direction at each of these critical points is towards a non-constant massless embedding $\theta(z)$. The critical values $2 \mu R=2 \ell+1$, indicate that the modes condensing are fermion bilinears which have R-charge 2 and are made from elementary fermion harmonics carrying energy $\left(\ell+\frac{1}{2}\right) R^{-1}$. The mass to charge ratio of the bilinears is $\left(\ell+\frac{1}{2}\right) R^{-1}$ and governs the critical value of $\mu R$ for the onset 
of a given instability. Schematically, the operator $\tilde{\psi}_{q i} \psi_{q}^{i}$ can be expressed as a sum over spherical harmonics, whose homogeneous mode on $S^{3}$ has the form,

$$
\frac{1}{2 \pi^{2}} \int d^{3} \Omega \tilde{\psi}_{q i} \psi_{q}^{i}=\sum_{\ell, n} \tilde{\psi}_{\ell, n}^{i} \psi_{\ell,-n}^{i}
$$

with $\psi_{\ell, n}$ an eigenmode of the Dirac operator on $S^{3}$ with mass $\left(\ell+\frac{1}{2}\right) R^{-1}, \ell=1,2, \ldots$ and $n$ denotes the azimuthal quantum numbers associated to a given harmonic. Therefore, the instabilities are triggered when the chemical potential times the Rcharge exceeds the mass of the composite charged scalar $\mathcal{O}_{\ell} \sim \sum_{n} \tilde{\psi}_{\ell, n} \psi_{\ell,-n}$.

The unstable directions seen above are also qualitatively distinct from the runaway behaviour encountered in $\mathcal{N}=4$ SYM for large enough R-charge 26, 27, 28 where the effect is due to the presence of flat directions for R-charged scalars. In that case, the scalar "Coulomb branch" instability is also visible at weak coupling. In the present setup, the (axial) R-charge is carried by the flavour fermions only. In the weakly interacting theory, when the chemical potential hits half-integer values, the chiral fermion number jumps and there is no instability (see Section(3)). In contrast, the elementary fermions are not visible in the strongly interacting limit; instead condensates of fermion bilinears are preferred when the chemical potential approaches the same half-integer points. At strong coupling these values are naturally interpreted as the mass to charge ratios of fermion bilinear modes. Surprisingly, the DBI action, at least within our ansatz, does not appear to include the end-point of this instability. Below we speculate on possible resolutions of this.

\section{Analytical results: instabilities}

We now turn to an analytical approach to reveal the origin of the instabilities we have encountered above, and in the process gain some understanding of the nature of the numerical solutions. The main features of the numerical approach, such as the appearance of new albeit unstable classical solutions, are a consequence of the nonlinearities of the DBI action. However, we expect some quantitative aspects to be accessible in a linearized regime, especially for large $\mu$. This can be understood to be a consequence of Eq.(4.17), which requires that for large enough $\mu R$, the amplitude of $\theta(z)$ is essentially bounded by $1 /(2 \mu R)$. The metastabilities and instabilities we saw above appear at an infinite set of values $2 \mu R=3,5,7, \ldots$, and so we expect some of this physics to be accessible in the linearized regime.

\subsection{Non-constant massless embeddings}

The first issue we would like to understand, is the appearance of a new saddle point or solution to the equations of motion with a finite density. When $\mu \gg 1, \sin \theta(z)<$ 
$(2 \mu)^{-1} \ll 1$, and it is then consistent to linearize the DBI equations of motion:

$$
\theta^{\prime \prime}(z)-\frac{48+16 z^{2}+5 z^{4}}{z\left(16-z^{4}\right)} \theta^{\prime}(z)+\frac{48+3 z^{4}+8 z^{2}\left(3+8 \mu^{2}\right)}{z^{2}\left(4+z^{2}\right)^{2}} \theta(z)=0 .
$$

As we will see more explicitly in the next section when we compute the two-point function for fluctuations around the $\theta=0$ solution, this is the equation of motion for a charged scalar coupled to a constant background gauge field in global AdS spacetime. It turns out that the equation can be exactly solved in terms of hypergeometric functions. This is somewhat easier to see after the change of variables

$$
z=2 \sqrt{y}, \quad \varphi(t) \equiv \frac{\theta(\sqrt{y})}{f(y)}, \quad f(y) \equiv(y-1) \sqrt{y-y^{-1}},
$$

following which we obtain a Schrödinger equation for $\varphi(y)$,

$$
\frac{d^{2} \varphi}{d y^{2}}+\left(\frac{\left(4 \mu^{2}-1\right)\left(y^{2}+1\right)}{y\left(y^{2}-1\right)^{2}}-\frac{8 \mu^{2}+1}{\left(y^{2}-1\right)^{2}}\right) \varphi=0 .
$$

The equation is solved by regularized hypergeometric functions

$$
\begin{aligned}
\theta(z) & =C_{1} \sqrt{y} \frac{(1+y)^{2 \mu}}{(1-y)^{2 \mu+1}}{ }_{2} \tilde{F}_{1}\left(\mu-\frac{1}{2}, \mu+\frac{1}{2} ; 2 \mu+1 ;\left(\frac{1+y}{1-y}\right)^{2}\right) \\
& +C_{2} \sqrt{y} \frac{(1-y)^{2 \mu-1}}{(1+y)^{2 \mu}}{ }_{2} \tilde{F}_{1}\left(-\mu-\frac{1}{2},-\mu+\frac{1}{2} ; 1-2 \mu ;\left(\frac{1+y}{1-y}\right)^{2}\right)
\end{aligned}
$$

where the coefficients are determined by requiring regularity at the origin of AdS spacetime at $z=2$ or $y=1$. Specifically, we are interested in embeddings that fill $A d S_{5}$ and get to the origin of the space, satisfying

$$
\left.\theta(z)\right|_{z=2}=\Upsilon,\left.\quad \theta^{\prime}(z)\right|_{z=2}=0
$$

Imposing these boundary conditions we find

$$
C_{1}=i \frac{4 \Upsilon e^{i \pi \mu}}{2 \mu-1} \frac{\Gamma\left(\frac{1}{2}+\mu\right)^{2}}{\pi \tan (\pi \mu)}, \quad C_{2}=C_{1} e^{-2 i \pi \mu} \frac{2 \mu-1}{2 \mu+1} \frac{\Gamma\left(\frac{1}{2}-\mu\right)^{2}}{\Gamma\left(\frac{1}{2}+\mu\right)^{2}} .
$$

It is readily checked that the above linear combination yields a real function ${ }^{7}$ which is regular at $z=2$. Near the AdS boundary $(z \rightarrow 0)$, we can then read off the coefficients in the asymptotic expansion (4.13). After reinstating the radius $R$ of the boundary three sphere which was effectively set to 1 in our conventions, we obtain

\footnotetext{
${ }^{7}$ To check the $z \rightarrow 2$ asymptotics and reality of the solution, one must choose the correct branch of the hypergeometic function which has a branch point at $z=2$ (when its argument diverges).
} 
the following analytic expressions for the mass $m$ and the condensate $\left\langle\tilde{\psi}_{q} \psi_{q}\right\rangle$

$$
\begin{aligned}
\theta_{(0)} & =-\Upsilon \frac{8}{\pi} \frac{\cos \pi \mu R}{4 \mu^{2} R^{2}-1}, \\
\theta_{(2)} & =-\Upsilon \frac{\cos \pi \mu R}{\pi\left(4 \mu^{2} R^{2}-1\right)} \times \\
& \times\left[3-\left(4 \mu^{2} R^{2}-1\right)\left\{\psi\left(\frac{1}{2}-\mu R\right)+\psi\left(\frac{1}{2}+\mu R\right)+\ln 4+2 \gamma_{E}-1\right\}\right] .
\end{aligned}
$$

Here $\psi(x)$ is the digamma function which has simple poles at $x=-n$, for all $n \in \mathbb{Z}$. Notice that in the expression for $\theta_{(2)}$ above, these poles are precisely cancelled by the zeroes of the cosine and $\theta_{(2)}$ is finite for all values of $\mu$.

Importantly, the set of linearized solutions, parametrized by $\Upsilon$, yields massless hypermultiplets precisely when

$$
\theta_{0}=0 \Longrightarrow 2 \mu R=2 \ell+1, \quad \ell=1,2,3, \ldots
$$

Therefore, non-constant massless embeddings first appear for half-integer values of the R-charge chemical potential. This is in agreement with the numerical results from the complete DBI action, which exhibit a more intricate structure including metastable and unstable regions in the vicinity of the same critical values. It is also easily checked that for these massless embeddings the chiral condensate is non-zero:

$$
\left\langle\tilde{\psi}_{q} \psi_{q}\right\rangle=\Upsilon \frac{\lambda N N_{f}}{2(2 \pi)^{4} R^{3}}(-1)^{\ell} .
$$

To summarize, the large $\mu$ linear analysis has shown that a continuous family of massless configurations, parametrized by $\Upsilon \leq \sin ^{-1}(1 / 2 \mu)$ and carrying a finite $\mathrm{R}$ charge density, emerges at the half-integer values of $\mu R$ indicated above. In contrast, the non-linear DBI equations show a similar set of (unstable) solutions spread out in a small window in the vicinity of these critical values.

The analytical expressions for the mass and the chiral condensate in Eq.(5.8), reproduce quite closely the numerical results displayed in the previous sections (see Fig.(9)).

\subsection{Stability analysis: two-point function for $\tilde{\psi}_{q} \psi_{q}$}

The detailed numerical study of the free energies of the various D7-brane embeddings, summarized in Fig.(8) suggests that the values $2 \mu R=3,5,7, \ldots$ are critical points where two saddle points of the free energy merge. We expect that small fluctuations around such points should cost no free energy at linear order, which is essentially what our linearized analysis above reveals. If the chemical potential were to be increased beyond these critical values, the system should become thermodynamically unstable. 

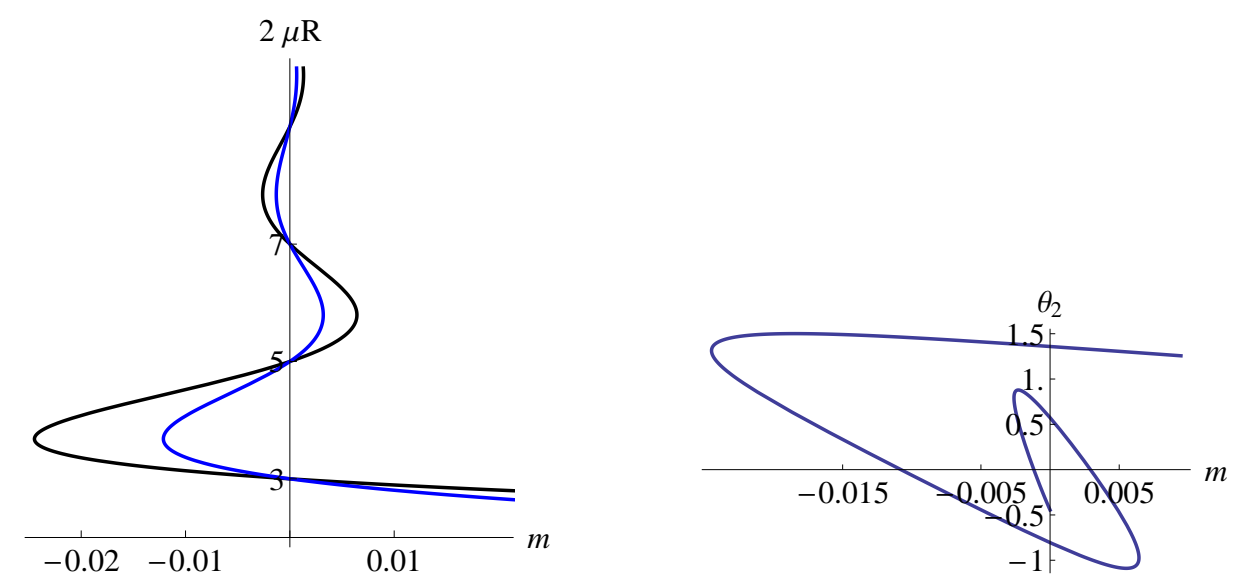

Figure 9: Plots of the expressions, obtained in the linearized approximation.

The natural object to compute, to look for the source of instabilities in the system is the two-point function of the operator dual to the slipping mode. In particular, the location of the poles in the frequency-space correlator of $\tilde{\psi}_{q} \psi_{q}$ will reveal the presence of instabilities in the system. Actually, the linearized analysis above can naturally be adapted to allow for a holographic evaluation of the two point function of the operator dual to $\theta$.

Since the dual field theory is formulated on the three-sphere, it is natural to consider temporal correlations of the $s$-wave mode of $\mathcal{O} \equiv \tilde{\psi}_{q} \psi_{q}$ :

$$
\mathcal{G}(t)=\iint \frac{d^{3} \Omega}{\operatorname{Vol}\left(\mathrm{S}^{3}\right)} \frac{d^{3} \Omega^{\prime}}{\operatorname{Vol}\left(\mathrm{S}^{3}\right)}\left\langle\mathcal{O}(t, \Omega) \mathcal{O}\left(0, \Omega^{\prime}\right)\right\rangle
$$

We begin by expanding the DBI action to quadratic order in $\theta$, allowing for the slipping angle to be time dependent, so that $\theta=\theta(z, t)$. The induced metric on the D7-brane is then

$$
\begin{aligned}
\left.d s^{2}\right|_{\mathrm{D} 7} & =-\left(\frac{\left(1+\frac{1}{4} z^{2}\right)^{2}}{z^{2}}-4 \mu^{2} \sin ^{2} \theta-\dot{\theta}^{2}\right) d t^{2}+\left(z^{2} \theta^{\prime}(z)^{2}+1\right) \frac{d z^{2}}{z^{2}}+ \\
& +2 \dot{\theta} \theta^{\prime} d t d z+\frac{\left(1-\frac{1}{4} z^{2}\right)^{2}}{z^{2}} d \Omega_{3}^{2}+\cos ^{2} \theta d \tilde{\Omega}_{3}^{2}
\end{aligned}
$$

At quadratic order, the DBI action yields

$$
\begin{aligned}
& S_{\mathrm{DBI}} \simeq \\
& \left.2 \pi^{2} \mathcal{N}_{\mathrm{D} 7}\left[\int d t d z \frac{\left(1-\frac{1}{4} z^{2}\right)^{3}\left(1+\frac{1}{4} z^{2}\right)}{z^{5}}\left(z^{2} \theta^{\prime 2}-\frac{z^{2}}{\left(1+\frac{1}{4} z^{2}\right)^{2}}\left(\dot{\theta}^{2}+4 \mu^{2} \theta^{2}\right)-3 \theta^{2}\right)\right)\right]
\end{aligned}
$$

This is precisely the quadratic action for a charged scalar in global $A d S_{5}$ with $m_{\text {scalar }}^{2}=-3$, coupled to a constant, background gauge potential $2 \mu$. This is natural, given the interpretation of $\mu$ as a chemical potential for a global $U(1)$ symmetry 
in the boundary theory. The extra factor of 2 is the charge of the fermion bilinear under the $U(1)$ R-symmetry in the boundary. This type of system is also encountered in holographic superconductor models, where there is typically a dynamical bulk Maxwell field coupled to a charged scalar [11, 23], leading to a VEV for the latter. The non-trivial embeddings with an R-charged condensate appear to be of a similar nature albeit in global AdS spacetime without a black hole.

Upon Fourier transforming to frequency space,

$$
\tilde{\theta}(z, \omega)=\int_{-\infty}^{\infty} d t e^{-i \omega t} \theta(z, t)
$$

we note that at this order the effect of a non-zero temporal frequency is a simple shift,

$$
\mu^{2} \rightarrow 4 \mu^{2}+\omega^{2}
$$

and $\tilde{\theta}(z, \omega)$ satisfies the linearized equation of motion Eq. (5.1) after the above replacement. Therefore, for the holographic calculation of the frequency space propagator we can simply import the relevant results from the previous section and make the replacement $\mu \rightarrow \frac{1}{2} \sqrt{4 \mu^{2}+\omega^{2}}$. The near boundary expansion of $\tilde{\theta}(z, \omega)$,

$$
\left.\tilde{\theta}(z, \omega)\right|_{z \rightarrow 0} \simeq \Theta_{0}(\omega) z+\Theta_{2}(\omega) z^{3}+\frac{1}{2}\left(1-4 \mu^{2}\right) \Theta_{0}(\omega) z^{3} \ln z+\ldots
$$

can be determined precisely from our linearized analysis above. In particular, we already know that regularity at the origin of AdS space leads to a relation between $\Theta_{2}$ and $\Theta_{0}$, from Eq. 5.8 :

$$
\begin{aligned}
\Theta_{2}(\omega) & =\Theta_{0}(\omega) \times \\
& \times\left[3-\left(4 \mu^{2}+\omega^{2}-1\right)\left\{\psi\left(\frac{1-\sqrt{4 \mu^{2}+\omega^{2}}}{2}\right)+\psi\left(\frac{1+\sqrt{4 \mu^{2}+\omega^{2}}}{2}\right)+\ln 4+2 \gamma_{E}-1\right\}\right] .
\end{aligned}
$$

Substituting this into the linearized action above, we evaluate the action induced on the boundary,

$$
S_{\mathrm{bdry}}=\left.2 \pi^{2} \mathcal{N}_{\mathrm{D} 7} \int_{-\infty}^{\infty} \frac{d \omega}{2 \pi} \frac{1}{\epsilon^{3}} \tilde{\theta}(\epsilon, \omega) \tilde{\theta}^{\prime}(\epsilon,-\omega)\right|_{\epsilon \rightarrow 0}+\ldots
$$

where we have not explicitly written out the counterterms. Differentiating twice with respect to $\Theta_{0}(\omega)$ which acts as the source for $\tilde{\psi}_{q} \psi_{q}(\omega)$, results in the frequency space correlator. We write the result after re-introducing the radius $R$ of the boundary three-sphere and ignoring "contact" terms which are regular in $\omega$ :

$$
\tilde{\mathcal{G}}(\omega)=\mathcal{N}_{\mathrm{D} 7}\left(4 \mu^{2}+\omega^{2}-R^{-2}\right)\left[\psi\left(\frac{1}{2}-\frac{1}{2} R \sqrt{4 \mu^{2}+\omega^{2}}\right)+\psi\left(\frac{1}{2}+\frac{1}{2} R \sqrt{4 \mu^{2}+\omega^{2}}\right)\right] .
$$

Despite the appearance of square roots in this expression, the function only has isolated simple poles at

$$
\omega= \pm \sqrt{R^{-2}(2 \ell+1)^{2}-4 \mu^{2}}, \quad \ell=1,2, \ldots
$$



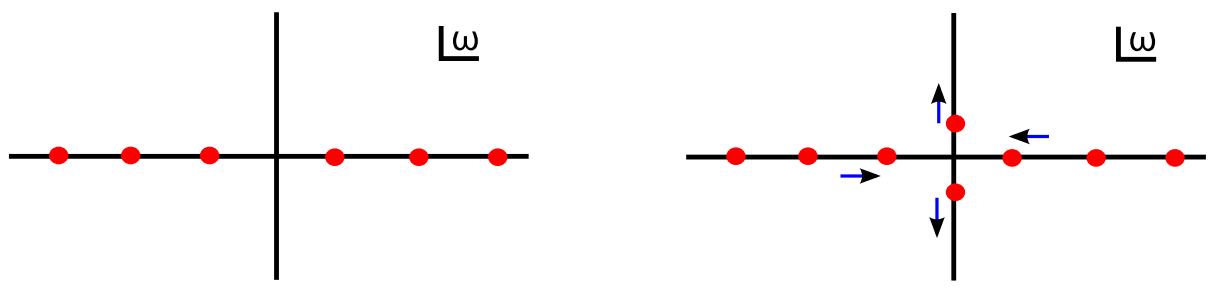

Figure 10: Simple poles of $\tilde{\mathcal{G}}(\omega)$ on the real axis, migrate to the imaginary axis as $\mu$ is increased past $\frac{3}{2} R^{-1}$.

For $\mu>\frac{3}{2} R^{-1}$, some of the poles move to the upper half plane, along the imaginary axis, reflecting an instability.

The $\mu=0$ functional form of the correlator coincides with the expression deduced from the weakly coupled field theory ${ }^{8}$ in Eq. (3.8). However, the normalizations differ between weak and strong couplings. Therefore the correlators do get renormalized even in the absence of a chemical potential.

In the strongly interacting system, the chemical potential has the effect of shifting the locations of the poles of the Green's function. With $\mu \neq 0$, there is a qualitative change in the physics in going to strong coupling. The discrete excitations at strong coupling consist of mesons/fermion bilinears made from the spherical harmonic states of elementary fermions. These composite scalars carry twice the R-charge of the fermions, and the poles of the correlator show that they possess twice the energy. Importantly, the R-charge chemical potential affects the masses of these states in the same way that it would alter the mass of a free complex scalar. A large enough chemical potential (bigger than the mass to charge ratio for these states) leads to a negative mass squared and triggers an instability favouring a condensate.

We expect that the instability persists when a flat space limit is taken, by formally letting $R$ become arbitrarily large, so that

$$
\tilde{\mathcal{G}}(\omega) \rightarrow \mathcal{N}_{\mathrm{D} 7}\left(\omega^{2}+4 \mu^{2}\right) \ln \left(\omega^{2}+4 \mu^{2}\right),
$$

which has non-analyticities in the upper half plane.

It is interesting to contrast the above picture with the physics of the free theory where the positions of the poles are unaltered by a non-vanishing chemical potential. The primary effect there is the Pauli blocking of low lying states, which in turn results in an imaginary part for the retarded Green's function in flat space beyond a kinematic threshold $|\omega|>2 \mu$ (Eq.(3.9)).

\section{D5 and D3' flavour branes in global $A d S_{5}$}

Most aspects of the analysis we performed for probe D7-branes above, carry over

${ }^{8}$ In recent work 38 the meson spectrum of probe branes in global AdS was obtained. The poles in the correlator we find here correspond to $l=\tilde{l}=0$ scalar states in that work. 
to probe D5 and D3-branes whose excitations in $A d S_{5}$ describe $2+1$ and $1+1$ dimensional defect CFTs coupled to $\mathcal{N}=4 \mathrm{SYM}$ at strong coupling [21, 22, 36]. In the global AdS context, the defect CFT's are formulated on a spatial $S^{2} \subset S^{3}$ and $S^{1} \subset S^{3}$. The eigenvalues of the Dirac operator on $S^{2}$ take integer values [41], so that

$$
\varepsilon_{\ell}\left(S^{2}\right)=\ell R^{-1}, \quad \ell=1,2, \ldots
$$

For the $1+1$ dimensional case, fermion energies on the $S^{1} \subset S^{3}$ are half-integer valued

$$
\varepsilon_{\ell}\left(S^{1}\right)=\left(\ell-\frac{1}{2}\right) R^{-1}, \quad \ell=1,2, \ldots
$$

Interestingly, the probe D-branes exhibit the same kind of instabilities we saw above for the D7-branes, but now at values of the chemical potential set by the fermion energy levels on $S^{2}$ and $S^{1}$. We will not repeat the detailed numerical study of these examples, since their basic features turn out to be quite similar to the probe D7brane. Instead, we will briefly explain the analytical results for the lower dimensional examples and point out features which are distinct from the four dimensional case.

\subsection{D5-brane probes}

The 2+1 dimensional CFT localized on the intersection of a large- $N$ number of D3-branes with $N_{f}$ D5-branes has eight supercharges and an $S U(2)_{H} \times S U(2)_{V}$ Rsymmetry. The defect theory has fundamental hypermultiplets, each containing a fermion doublet transforming under $S U(2)_{V}$. We wish to introduce a chemical potential for the $U(1)$ subgroup of this $S U(2)_{V}$ R-symmetry under which the fundamental representation fermions are charged. As shown in [21] the scalars in the fundamental hypermultimplets are uncharged under this. In the near horizon limit of this system at large- $N$, probe D5-branes in (the Poincare' patch of) $A d S_{5} \times S^{5}$ can therefore be chosen to spin along the internal directions so that R-charge is concentrated in the fermionic sector of the fundamental matter fields.

The same setup, when carried over to global AdS spacetime, involves flavour D5branes with world-volume global $A d S_{4} \times S^{2}$. The $S^{2} \subset S^{5}$ wrapped by the branes can be parametrized in terms of the angular coordinate $\theta$ as,

$$
d s_{S^{5}}^{2}=d \theta^{2}+\sin ^{2} \theta d \tilde{\Omega}_{2}^{2}+\cos ^{2} \theta d \Omega_{2}^{2} .
$$

$\theta$ plays the role of the "slipping" angle and the D5-branes can be chosen to spin along the azimuthal angle $\phi$ inside the transverse two-sphere $\tilde{S}^{2}$. The induced metric for the spinning D5-brane with world-volume $A d S_{4} \times S^{2}$ is

$$
\begin{aligned}
& \left.d s^{2}\right|_{\mathrm{D} 5}= \\
& -\left(\frac{\left(1+\frac{1}{4} z^{2}\right)^{2}}{z^{2}}-4 \mu^{2} \sin ^{2} \theta\right) d t^{2}+\left(z^{2} \theta^{\prime}(z)^{2}+1\right) \frac{d z^{2}}{z^{2}}+\frac{\left(1-\frac{1}{4} z^{2}\right)^{2}}{z^{2}} d \Omega_{2}^{2}+\cos ^{2} \theta d \Omega_{2}^{2} .
\end{aligned}
$$




\subsubsection{Linearized results:}

The D5-brane probe action, in terms of embedding coordinates $\left\{\xi_{i}\right\}$, is

$$
S_{\mathrm{D} 5}=N_{f} T_{\mathrm{D} 5} \int d^{5} \xi \sqrt{-\operatorname{det}^{*} g}, \quad \mathcal{N}_{\mathrm{D} 5} \equiv N_{f} T_{\mathrm{D} 3} 4 \pi=\frac{\sqrt{\lambda}}{4 \pi^{3}} N_{f} N
$$

and the resulting non-linear equations of motion can be numerically solved. Similarly to the D7-brane case, we look for embeddings of the slipping mode $\theta(z)$ in the linearized approximation. This mode maps to a dimension two R-charged operator in the boundary 3D CFT, namely the quark (fermion) bilinear $\tilde{\psi}_{q} \psi_{q}$. The solutions of interest are those that get to the origin of $A d S$. At quadratic order the spinning D5brane action describes a charged scalar in $A d S_{4}$, with $m_{\text {scalar }}^{2}=-2$, in the presence of a constant background gauge potential $2 \mu$. The linear equation of motion,

$$
\theta^{\prime \prime}(z)-2 \frac{16+4 z^{2}+2 z^{4}}{z\left(16-z^{4}\right)} \theta^{\prime}(z)+2 \frac{16+z^{4}+8 z^{2}\left(1+4 \mu^{2}\right)}{z^{2}\left(4+z^{2}\right)^{2}} \theta(z)=0
$$

subject to the boundary conditions,

$$
\left.\theta^{\prime}(z)\right|_{z=2}=0,\left.\quad \theta(z)\right|_{z=2}=\Upsilon
$$

is solved by

$$
\theta(z)=4 \Upsilon \frac{z}{\mu} \frac{\sin \left(4 \mu \tan ^{-1} \frac{z}{2}-\frac{\pi \mu}{2}\right)}{\left(z^{2}-4\right)}
$$

The asymptotic expansion near the boundary then yields the mass and the fermion bilinear condensate,

$$
\begin{aligned}
& \theta(z) \simeq \theta_{(0)} z+\theta_{2} z^{2}+\ldots \\
& \theta_{(0)}=\Upsilon \frac{\sin (\pi \mu R)}{\mu R}, \quad \theta_{(2)}=-\Upsilon \cos (\pi \mu R)
\end{aligned}
$$

Therefore the linearized embeddings are massless only when,

$$
\mu R=\ell R^{-1} \quad \ell=1,2,3, \ldots
$$

As before, these are critical values of the chemical potential beyond which corresponding fermion bilinears will become unstable, as we show below. For these finite density embeddings the chiral condensate is non-zero,

$$
\left\langle\tilde{\psi}_{q} \psi_{q}\right\rangle=-\mathcal{N}_{\mathrm{D} 5}(-1)^{\ell} \quad \ell \in \mathbb{Z}
$$




\subsubsection{Stability of $\theta=0$ embedding:}

The calculation of the $s$-wave 2-point function in frequency space, for the fermion bilinear $\mathcal{O}=\tilde{\psi}_{q} \psi_{q}$ proceeds exactly as in the $3+1$ dimensional theory. The result is obtained by taking the ratio of the normalizable and non-normalizable modes in (6.10), followed by the replacement $\mu \rightarrow \frac{1}{2} \sqrt{4 \mu^{2}+\omega^{2}}$. We find,

$$
\tilde{\mathcal{G}}(\omega)=\mathcal{N}_{\mathrm{D} 5} \sqrt{4 \mu^{2}+\omega^{2}} \cot \left(\frac{\pi}{2} R \sqrt{4 \mu^{2}+\omega^{2}}\right)
$$

which has simple poles at

$$
\omega= \pm \sqrt{(2 \ell)^{2} R^{-2}-4 \mu^{2}}, \quad \ell=1,2, \ldots .
$$

Therefore the onset of instabilities occurs when $\mu R>1$, when the poles move to the upper half plane. Once again the chemical potential acts as a negative mass squared for the scalar excitations at strong coupling

\section{2 $\mathrm{D3}^{\prime}$ probe branes}

The theory on the intersection of the D3-D3' system was studied in detail in 22]. In flat space, the D-brane setup involves two stacks with $N$ and $N_{f}$ D3-branes, spanning the $x^{0}, x^{1}, x^{2}, x^{3}$ and $x^{0}, x^{1}, x^{4}, x^{5}$ directions respectively. Each stack yields an $\mathcal{N}=4$ vector multiplet in four dimensions, coupled to $S U(N) \times S U\left(N_{f}\right)$ bifundamental degrees of freedom localized at the intersection of the two stacks.

In the 't Hooft large- $N$ limit with $N_{f}$ fixed, the $S U\left(N_{f}\right)$ vector multiplet decouples since its effective coupling $\lambda_{f} \equiv \lambda N_{f} / N$ is vanishing in the large- $N$ limit. Therefore, one obtains $\mathcal{N}=4$ SUSY Yang-Mills theory in four dimensions coupled to $N_{f}$ two dimensional $\mathcal{N}=(4,4)$ fundamental hypermultiplets localized at the intersection of the two sets of D3-branes. In this limit, and at strong 't Hooft coupling, the embedding of the $N_{f}$ probe D $3^{\prime}$-branes in the $A d S_{5} \times S^{5}$ geometry induces an $A d S_{3} \times S^{1}$ metric on the probes. The $S^{1} \subset S^{5}$, wrapped by the D $3^{\prime}$ s can be specified as usual by the slipping angle $\theta$

$$
d \Omega_{5}^{2}=d \theta^{2}+\sin ^{2} \theta d \Omega_{3}^{2}+\cos ^{2} \theta d \Omega_{1}^{2} .
$$

The global isometry of the coordinates transverse to all the D3-branes is $S O(4) \simeq$ $S U(2)_{L} \times S U(2)_{R}$. Under this isometry, the fermions in the fundamental $(4,4)$ hypermultiplets transform in the $\left(\frac{1}{2}, 0\right)$ and $\left(0, \frac{1}{2}\right)$ representations respectively. Their scalar superpartners are uncharged under this. The fermion bilinear, which we will continue to denote schematically as $\tilde{\psi}_{q} \psi_{q}$, dual to the slipping mode, transforms in the $\left(\frac{1}{2}, \frac{1}{2}\right)$ representation of $S U(2)_{L} \times S U(2)_{R}$. It has scaling dimension $\Delta=1$,

consistent with a fermion bilinear in two dimensions, and is a $\frac{1}{2}$-BPS operator.

The above system in global AdS spacetime is dual to the $1+1$ dimensional defect CFT formulated on an equatorial circle of the boundary three-sphere. Imparting an 
R-charge to the flavour fermions by rotating the D3's along an angular coordinate $\phi$ in the $S^{3}$ with angular velocity $2 \mu$, the induced metric on the D3' becomes

$$
\begin{aligned}
& \left.d s^{2}\right|_{\mathrm{D} 3^{\prime}}= \\
& -\left(\frac{\left(1+\frac{1}{2} z^{2}\right)^{2}}{z^{2}}-4 \mu^{2} \sin ^{2} \theta\right) d t^{2}+\left(z^{2} \theta^{\prime}(z)^{2}+1\right) \frac{d z^{2}}{z^{2}}+\frac{\left(1-\frac{1}{2} z^{2}\right)^{2}}{z^{2}} d \Omega_{1}^{2}+\cos ^{2} \theta d \Omega_{1}^{2}
\end{aligned}
$$

This yields the probe D-brane action

$$
S_{\mathrm{D}^{\prime}}=N_{f} T_{\mathrm{D} 3} \int d^{4} \xi \sqrt{-\operatorname{det}^{*} g}, \quad T_{\mathrm{D}^{\prime}}=\frac{N}{2 \pi^{2}} .
$$

The slipping mode, at linearized order, is a scalar with $m_{\text {scalar }}^{2}=-1$ in $A d S_{3}$. It therefore saturates the relevant Breitenlohner-Freedman bound with boundary asymptotics,

$$
\theta(z) \rightarrow \theta_{(0)} z \ln z+\theta_{(2)} z+\ldots
$$

Unlike the higher dimensional cases, the term linear in $z$ is now normalizable, whilst the non-normalizable mode is the coefficient of the asymptotic logarithmic term. The latter plays the role of the hypermultiplet mass. When $\mu \gg 1$, it is consistent to linearize the DBI equation of motion,

$$
\theta^{\prime \prime}(z)-\frac{\left(16+3 z^{4}\right)}{z\left(16-z^{4}\right)} \theta^{\prime}(z)-\frac{16+8 z^{2}\left(1+8 \mu^{2}\right)+z^{4}}{z^{2}\left(4+z^{2}\right)^{2}} \theta(z)=0 .
$$

The solutions to this equation, which fall in the category of "AdS-filling" embeddings, can be written in terms of hypergeometric functions satisfying the boundary conditions,

$$
\left.\theta(z)\right|_{z=2}=\left.\Upsilon \quad \theta^{\prime}(z)\right|_{z=2}=0 .
$$

The corresponding solution is

$$
\begin{aligned}
& \theta(z)=-\Upsilon \frac{i}{2} \frac{\sqrt{y-1}}{\sin \pi \mu}\left(\frac { e ^ { i \pi \mu } y ^ { \mu } \Gamma ( \frac { 1 } { 2 } + \mu ) } { \Gamma ( \frac { 1 } { 2 } - \mu ) \Gamma ( 1 + 2 \mu ) } { } _ { 2 } F _ { 1 } \left(\frac{1}{2}+\mu, \frac{1}{2}+\mu ; 1+2 \mu ; y(\phi .21)\right.\right. \\
& y \equiv \frac{\left(1+\frac{1}{4} z^{2}\right)^{2}}{\left(1-\frac{1}{4} z^{2}\right)^{2}} .
\end{aligned}
$$

From the asymptotic expansion of this function, we read off the fermion mass and condensate

$$
\begin{aligned}
& \theta_{(0)}=-\frac{2}{\pi} \Upsilon \cos (\pi \mu R), \\
& \theta_{(2)}=-\frac{1}{\pi} \Upsilon \cos (\pi \mu R)\left[\psi\left(\frac{1}{2}+\mu R\right)+\psi\left(\frac{1}{2}-\mu R\right)+2 \gamma_{E}+\ln 4\right] .
\end{aligned}
$$


As for probe D5 and D7-branes, the VEV and the mass satisfy a relation

$$
\theta_{(2)}=\frac{1}{2} \theta_{(0)}\left[\psi\left(\frac{1}{2}+\mu R\right)+\psi\left(\frac{1}{2}-\mu R\right)+2 \gamma_{E}+\ln 4\right] .
$$

Therefore the embeddings yield zero mass hypermultiplets when

$$
2 \mu R=2 \ell-1, \quad \ell=1,2,3, \ldots,
$$

which is in agreement with what we would expect for fermion bilinears on $S^{1}$. To correctly calculate both one- and two-point functions of the operator $\tilde{\psi}_{q} \psi_{q}$, it is useful to recall the holographic renormalization procedure for probe branes [34] applied to the D3-D3' system. This is particularly relevant in this case due to putative logarithmic divergences in $1+1$ dimensions. The regularized DBI action is obtained by performing a subtraction at $z=\epsilon \ll 1$,

$$
S_{\mathrm{D} 3^{\prime}}^{\mathrm{reg}}=4 \pi^{2} N_{f} T_{\mathrm{D} 3^{\prime}} \int d t\left(\int_{\epsilon}^{2} d z \sqrt{-\operatorname{det}^{*} g}-\frac{1}{2 \epsilon^{2}}+\frac{1}{2 \epsilon^{2}} \theta(\epsilon, t)^{2}\left(1+\frac{1}{\ln \epsilon}\right)\right)
$$

Using this, it follows that

$$
\left\langle\tilde{\psi}_{q} \psi_{q}\right\rangle=\lim _{\epsilon \rightarrow 0} \frac{\ln \epsilon}{\epsilon} \frac{1}{\sqrt{-\gamma}} \frac{\delta S_{\mathrm{D} 3^{\prime}}^{\mathrm{reg}}}{\delta \theta(\epsilon, t)}=\frac{N N_{f}}{2 \pi R} \theta_{(2)},
$$

where we have restored explicit dependence on the radius of the boundary circle. Therefore for the massless, finite density embeddings in the vicinity of $2 \mu R=2 \ell-1$ in the linearized approximation, the chiral condensate is

$$
\left.\left\langle\tilde{\psi}_{q} \psi_{q}\right\rangle\right|_{m=0}=\Upsilon \frac{N N_{f}}{2 \pi R}(-1)^{\ell}, \quad \ell \in \mathbb{Z}
$$

\subsubsection{Two-point function around $\theta=0$ :}

It is now straightforward to obtain the two-point function of the fluctuations of $\mathcal{O}=\tilde{\psi}_{q} \psi_{q}$, about the trivial zero-density embedding in frequency space. A time dependent fluctuation $\theta(z, t)$ about the $\theta=0$ solution, can be expanded near $z=0$ as (in frequency space),

$$
\tilde{\theta}(z, \omega)=\Theta_{0}(\omega) z \ln z+\Theta_{2}(\omega) z+\ldots
$$

The VEV of $\mathcal{O}(\omega)$ and the source $\Theta_{0}(\omega)$ obey the relation (6.23) with the replacement $\mu \rightarrow \frac{1}{2} \sqrt{4 \mu^{2}+\omega^{2}}$. The Green's function for $\mathcal{O}$ is then given by

$$
\begin{aligned}
& \langle\mathcal{O}(\omega) \mathcal{O}(-\omega)\rangle=-\frac{1}{2} \frac{\delta\langle\mathcal{O}(\omega)\rangle}{\delta \Theta_{0}(-\omega)} \\
& =-\frac{N N_{f}}{4 \pi R}\left[\psi\left(\frac{1}{2}+\frac{1}{2} R \sqrt{4 \mu^{2}+\omega^{2}}\right)+\psi\left(\frac{1}{2}-\frac{1}{2} R \sqrt{4 \mu^{2}+\omega^{2}}\right)+2 \gamma_{E}+\ln 4\right] .
\end{aligned}
$$


As was noted in [22], the Green's functions and indeed, the DBI action associated to probe D3-branes (6.17) is independent of the 'tHooft coupling $\lambda$, suggesting a non-renormalization theorem. However, this could only possibly be true at $\mu=0$, in the supersymmetric theory. In the situation with a chemical potential we see that the locations of poles in the correlator depend non-trivially on $\mu$. In particular, as in the higher dimensional examples, the poles of the Green's function shift to

$$
\omega= \pm \sqrt{(2 \ell-1)^{2} R^{-2}-4 \mu^{2}}, \quad \ell=1,2,3, \ldots
$$

which imply instabilities when $\mu R>\frac{1}{2}$.

\section{Discussion}

We have seen that spinning Dp-branes in global $A d S_{5} \times S^{5}$, dual to strongly coupled CFT's on spheres with a chemical potential for fermion flavours alone, exhibit instabilities (preceded by metastabilities). It appears that in all cases the zero density state becomes metastable or unstable when the chemical potential approaches integer or half-integer values, depending on the number of spatial dimensions in the boundary CFT. At each of these points, the systems would prefer to be in a configuration with finite $\mathrm{R}$-charge density and with a homogeneous condensate made from harmonics of the elementary fermions on the spheres.

However, the probe DBI analysis leaves the central question unanswered, namely, what is the end-point of the unstable directions. The answer to this is not clear from the above computations, since the fully non-linear DBI solutions show a runaway effective potential. One plausible explanation could be that determining the true ground state might require new active "light" modes on the probe brane. This could happen, for example, if the ground state were inhomogeneous, as is the case in certain lower dimensional models 39

However, a possible answer may lie in the differing, and somewhat complementary, physical pictures that emerge at arbitrarily weak or zero coupling and strong coupling. In the free theory, the relevant degrees of freedom are the elementary fermions (and their spherical harmonics), whereas in the strongly interacting AdS/CFT dual, the D-brane "slipping mode" is the natural degree of freedom. This is a composite scalar - a fermion bilinear. A large enough R-symmetry chemical potential, effectively induces a negative mass squared for this composite scalar. This situation is reminiscent of the Gross-Neveu model [40] where the elementary fermions are traded for a composite scalar and an effective potential for this composite scalar is subsequently induced, leading to chiral symmetry breaking. In that case, the full effective potential requires resumming fermion (flavour) loops. It is conceivable that in the present context similar, subleading corrections involving fermion loops need to be incorporated into the picture. This would also potentially mean inclusion of 
back-reaction effects of flavour branes on the background geometry. The possibility remains, of approaching this question from the weakly coupled boundary theory where effective four-fermi interactions can be induced via the Yukawa couplings of the flavour fermions to the adjoint modes of the $\mathcal{N}=4$ theory on the sphere.

Acknowledgements: I would like to thank Gert Aarts, Paolo Benincasa, Simon Hands, Tim Hollowood, Andreas Karch, Andy O' Bannon, Jim Rafferty and Larry Yaffe for discussions and useful comments. Special thanks to Andy O' Bannon and Paolo Benincasa for insightful comments and suggestions on early drafts of the manuscript. I also acknowledge the stimulating atmosphere provided by the Erwin Schrödinger Institute, during the EMMI Workshop on "Hot Matter: Quasiparticles or Quasinormal modes", August 2010, where some of this study was initiated. This work is supported by STFC Rolling Grant ST/G0005006/1. 


\section{Appendix A: Weak coupling Gross-Witten transitions}

In [12], it was shown that large- $N$ Yang-Mills theory on $S^{3}$ with $N_{f}$ fermions in the fundamental representation $\left(N_{f} / N\right.$ fixed), undergoes an infinite sequence of GrossWitten transitions as a function of a baryon number chemical potential, at a fixed low temperature. In that case, the Euclidean path integral was shown to be dominated by a complexified saddle point configuration of eigenvalues of the Polyakov-loop at large$N$. The complex nature of the saddle point was due to the fact that the Euclidean action with a baryon number chemical potential, is not Hermitian - the source of the "sign problem".

Below, we will repeat the essential aspects of this computation for the case where a chemical potential is introduced for an axial $U(1)$ symmetry for the fermions. We take $N_{f}$ flavours of massless Dirac fermions

$$
\psi_{D}^{i}=\left(\begin{array}{c}
\psi_{q}^{i} \\
\tilde{\psi}_{q}^{i \dagger}
\end{array}\right) \quad i=1,2, \ldots N_{f}
$$

In Lorentzian signature, a chemical potential $\mu$ for the axial $U(1)$ symmetry, is introduced by

$$
\mathcal{L} \rightarrow \mathcal{L}+\mu \sum_{i=1}^{N_{f}} \bar{\psi}_{D i} \gamma^{0} \gamma_{5} \psi_{D}^{i}+\ldots
$$

where the "..." represent terms due to any additional matter fields in the theory charged under the $U(1)$. To make sense of this we need to assume that the symmetry is anomaly free, which can be achieved in supersymmetric theories with an appropriately chosen matter content (e.g. the matter content of $\mathcal{N}=2$ supersymmetric gauge theory with $S U(N)$ gauge group and $N_{f}=2 N$ hypermultiplets). The main feature we want to illustrate is largely independent of these details.

At finite temperature $T$, going to Euclidean signature, the theory is formulated on $S^{3} \times S^{1}$. The circumference of the $S^{1}$ is $\beta=1 / T$. Allowing for a Wilson loop around the thermal circle, we can choose it to be diagonal and homogeneous on $S^{3}$

$$
\frac{1}{\operatorname{Vol}\left(S^{3}\right)} \oint_{S^{1} \times S^{3}} A_{0}=\operatorname{diag}\left(\theta_{1}, \theta_{2}, \ldots \theta_{N}\right), \quad \sum_{i=1}^{N} \theta_{i}=0 \bmod 2 \pi
$$

Fermions do not have any zero modes on $S^{3}$ and at finite temperature. Assuming that any other matter fields in the theory do not have zero modes, we can integrate out all the Kaluza-Klein harmonics on $S^{3}$ to obtain an effective unitary matrix model for the Polyakov loop $U \equiv \exp i \oint_{S^{1}} A_{0}$. In the eigenvalue basis, (A.3) the effective 
action for the $\theta_{i}$ takes the form [30, 28, 26, 12]

$$
\begin{aligned}
& S_{\text {eff }}\left[\theta_{i}\right]=\sum_{i, j=1}^{N}-\frac{1}{2} \ln \sin ^{2}\left(\frac{\theta_{i}-\theta_{j}}{2}\right)-\frac{N_{f}}{2} \sum_{j=1}^{N} d_{\ell} \ln \left(1+e^{i \theta_{j}} e^{-\beta\left(\varepsilon_{\ell}-\mu\right)}\right) \\
& -\frac{N_{f}}{2} \sum_{j=1}^{N} d_{\ell} \ln \left(1+e^{-i \theta_{j}} e^{-\beta\left(\varepsilon_{\ell}-\mu\right)}\right)-(\mu \rightarrow-\mu)+\ldots
\end{aligned}
$$

The first term is the Van der Monde pairwise repulsive potential between the eigenvalues, while the "..." represent the contributions from Kaluza-Klein harmonics of all other degrees of freedom in the theory. The terms explicitly shown arise from integrating out the fermion spherical harmonics, transforming in the fundamental representation of $S U(N)$. The energies of the fermion modes and their degeneracies on $S^{3}$ are

$$
\varepsilon_{\ell}=\ell+\frac{1}{2} \quad d_{\ell}=2 \ell(\ell+1), \quad \ell=0,1,2 \ldots
$$

We note that the effective action is real, in contrast to the situation with baryonic chemical potential [28]; nevertheless, the physics is quite similar to that case.

The physics of interest occurs at low $T$, when $\mu$ approaches $\varepsilon_{\ell}$. When $\mu \simeq \varepsilon_{\ell}$, it suffices to focus attention solely on the mode with that energy. In this regime it is useful to introduce the fugacity

$$
\begin{array}{rlrl}
\zeta_{\ell} \equiv e^{-\left(\varepsilon_{\ell}-\mu\right) / T} & \zeta_{\ell} \ll 1 & \text { for } \quad \mu \lesssim \varepsilon_{\ell}, \\
& \zeta_{\ell} \gg 1 \text { for } \quad \mu \gtrsim \varepsilon_{\ell},
\end{array}
$$

In the large- $N$ limit with $N_{f} \rightarrow \infty$ and $N_{f} / N$ fixed, the saddle point equation for the effective action with $\mu \simeq \varepsilon_{\ell}$, is

$$
\sum_{j=1}^{N} \frac{1}{N} \cot \left(\frac{\theta_{i}-\theta_{j}}{2}\right) \simeq \frac{N_{f}}{N} d_{\ell} \frac{\zeta_{\ell} \sin \theta}{\zeta_{\ell}^{2}+2 \zeta_{\ell} \cos \theta+1}+\text { exponentially small }
$$

When $\mu<\varepsilon_{\ell}, \zeta_{\ell} \ll 1$ and the saddle point equation becomes

$$
\int_{-\pi}^{\pi} d \theta^{\prime} \rho\left(\theta^{\prime}\right) \cot \left(\frac{\theta-\theta^{\prime}}{2}\right) \simeq d_{\ell} \frac{N_{f}}{N} \zeta_{\ell} \sin \theta, \quad \zeta_{\ell}<1 .
$$

while for $\mu>\varepsilon_{\ell}$

$$
\int_{-\pi}^{\pi} d \theta^{\prime} \rho\left(\theta^{\prime}\right) \cot \left(\frac{\theta-\theta^{\prime}}{2}\right) \simeq d_{\ell} \frac{N_{f}}{N} \frac{1}{\zeta_{\ell}} \sin \theta, \quad \zeta_{\ell}>1 .
$$

Here we have introduced the eigenvalue density $\rho(\theta)$ on the circle, normalized so that $\int_{-\pi}^{\pi} d \theta \rho(\theta)=1$. These are precisely the equations leading to the Gross-Witten third order transition, [31. 
This analysis, (from the results of [31]) shows that for both $\mu<\varepsilon_{\ell},\left(\zeta_{\ell} \ll 1\right)$ and $\mu>\varepsilon_{\ell},\left(\zeta_{\ell} \gg 1\right)$, the distribution function $\rho(\theta)$ should be "ungapped".

As $\mu$ is increased from low values towards $\varepsilon_{\ell}$, we expect a "gapping" transition of third-order when

$$
\frac{N_{f}}{N} d_{\ell} \zeta_{\ell} \simeq 1 .
$$

which corresponds to

$$
\mu=\mu_{-}^{*} \equiv \varepsilon_{\ell}-T \ln \left(\frac{N_{f}}{N} d_{\ell}\right),
$$

for small enough $T$. Therefore for $\mu>\mu_{-}^{*}$, the theory enters a gapped phase for the eigenvalues of the Polyakov loop.

As $\mu$ is increased further past $\varepsilon_{\ell}$, the effective potential becomes of the form (A.9) and now the theory undergoes yet another transition from the gapped to an ungapped phase at

$$
\begin{gathered}
\frac{N_{f}}{N} d_{\ell} \frac{1}{\zeta_{\ell}} \simeq 1 \\
\Longrightarrow \quad \mu=\mu_{+}^{*} \equiv \varepsilon_{\ell}+T \ln \left(\frac{N_{f}}{N} d_{\ell}\right) .
\end{gathered}
$$

Therefore, we learn that the free theory on $S^{3}$ at very low temperatures, experiences a pair of Gross-Witten transitions as a function of the chemical potential $\mu$, when the chemical potential is in the vicinity of an energy level of a fermionic mode on $S^{3}$.

We also expect intuitively that across these transitions, the level with energy $\varepsilon_{\ell}$ should become occupied. This can be verified by computing the derivative of the action/free energy with respect to $\mu$ :

$$
\left\langle\left(N_{L}-N_{R}\right)\right\rangle=-T \frac{\partial S_{\mathrm{eff}}}{\partial \mu}=N_{f} N \sum_{\ell} d_{\ell} \zeta_{\ell} \int d \theta^{\prime} \rho\left(\theta^{\prime}\right) \frac{\zeta_{\ell}+\cos \theta^{\prime}}{1+\zeta_{\ell}^{2}+2 \zeta_{\ell} \cos \theta^{\prime}}
$$

When $\mu<\varepsilon_{\ell}, \zeta_{\ell} \ll 1$ at low $T$, and therefore the corresponding energy level is unoccupied. For $\mu>\varepsilon_{\ell}$, on the other hand, since $\zeta_{\ell} \gg 1$, the corresponding energy level has occupation number,

$$
N_{f} N d_{\ell} \zeta_{\ell} \int d \theta^{\prime} \rho\left(\theta^{\prime}\right) \frac{\zeta_{\ell}+\cos \theta^{\prime}}{1+\zeta_{\ell}^{2}+2 \zeta_{\ell} \cos \theta^{\prime}} \approx N_{f} N d_{\ell} \quad \zeta_{\ell} \gg 1 .
$$

It is also interesting to note that in the ungapped phase, when $\rho(\theta) \approx \frac{1}{2 \pi}$, the Polyakov loop is

$$
\frac{1}{N}\langle\operatorname{Tr} U\rangle=\int_{-\pi}^{\pi} d \theta e^{i \theta} \rho(\theta) \approx 0, \quad \text { when } \zeta_{\ell} \gg 1 \text { or } \zeta_{\ell} \ll 1 .
$$

In the gapped phase, however, the Polyakov loop will have an expectation value of order one. Therefore, in addition to a jump in the (chiral) fermion occupation number 
when $\mu_{-}^{*} \lesssim \mu \lesssim \mu_{+}^{*}$, the Polyakov loop exhibits a spike across these transitons. The situation is illustrated in Fig.(3).

\section{Appendix B: Finite density propagators}

In this section we use real time fermion propagators at finite density to sketch the perturbative computation of the correlator of the operator $\tilde{\psi}_{q} \psi_{q}$. We begin by quoting the results for fermions in flat space [42, and modify them slightly to account for the fact that in our problem the chemical potential is for an axial $U(1)$ symmetry. We use the flat space results for the two-point function of $\tilde{\psi}_{q} \psi_{q}$ to infer its form on $S^{3}$. We package the fermion flavours in the D3-D7 setup into $N_{f}$ Dirac fermions as in Eq.(A.1) $\left\{\psi^{i}\right\}, i=1,2, \ldots N_{f}$. The momentum space Feynman propagator is defined via

$$
i S(\vec{k} ; t, 0) \delta_{m}^{l}=\int d^{3} x e^{-i \vec{k} \cdot \vec{x}}\left\langle\psi^{l}(\vec{x}, t) \bar{\psi}_{m}(0)\right\rangle .
$$

where $l, m=1,2 \ldots N_{f}$. The Feynman propagator can be written as the sum of retarded and advanced pieces

$$
S(\vec{k} ; t, 0)=S^{>}(\vec{k}, t, 0) \Theta(t)+S^{<}(\vec{k} ; t, 0) \Theta(-t) .
$$

For the case with an axial $U(1)$ chemical potential, we write

$$
S^{>(<)}(\vec{k} ; t, 0)=\frac{1-\gamma_{5}}{2} S_{-}^{>(<)}(\vec{k} ; t, 0)+\frac{1+\gamma_{5}}{2} S_{+}^{>(<)}(\vec{k} ; t, 0) .
$$

With massless, chirality "plus" fermions, $k^{\nu} \equiv(k, \vec{k})$, we have

$$
\begin{aligned}
& S_{+}^{>}(\vec{k} ; t, 0)=-\frac{i}{2 k}\left(k\left[1-n_{F}(k, \mu)\right] e^{-i k t}+\gamma_{0} k \gamma_{0} \bar{n}_{F}(k, \mu) e^{i k t}\right) \\
& S_{+}^{<}(\vec{k} ; t, 0)=\frac{i}{2 k}\left(k n_{F}(k, \mu) e^{-i k t}+\gamma_{0} k \gamma_{0}\left[1-\bar{n}_{F}(k, \mu)\right] e^{i k t}\right) .
\end{aligned}
$$

The right-handed fermion propagators $S_{-}^{>}$and $S_{-}^{<}$are the same as above with the replacement $\mu \rightarrow-\mu$. The Fermi-Dirac distributions which turn into step functions at zero temperature are given as

$$
\begin{aligned}
& n_{F}(k, \mu)=\frac{1}{e^{\beta(k-\mu)}+1} \rightarrow \Theta(\mu-k) \quad \text { as } \quad T \rightarrow 0, \\
& \bar{n}_{F}(k, \mu)=\frac{1}{e^{\beta(k+\mu)}+1} \rightarrow \Theta(-\mu-k) \text { as } \quad T \rightarrow 0 .
\end{aligned}
$$

We are interested in computing the spatially homogeneous (zero external momentum) part of the two-point function for $\mathcal{O}$,

$$
\mathcal{O} \equiv \sum_{i=1}^{N_{f}} \tilde{\psi}_{q i} \psi_{q}^{i}, \quad \mathcal{G}(t) \equiv \int d^{3} x\langle\mathcal{O}(\vec{x}, t) \mathcal{O}(0)\rangle
$$




\section{Time-ordered propagator:}

It is straightforward to show that

$$
\mathcal{G}(t)=N_{f} \int \frac{d^{3} k}{(2 \pi)^{3}} \operatorname{Tr}[S(\vec{k} ; 0, t) S(\vec{k} ; t, 0)]
$$

where the trace is over Dirac and colour indices, and all propagators are time ordered. On $S^{3}$, the integral over spatial momenta should be replaced by a discrete sum over the spherical harmonic index $\ell$ with degeneracy $2 \ell(\ell+1)$. The Feynman propagator for $\mathcal{O}$ is then,

$$
\mathcal{G}(t)=N_{f} \int \frac{d^{3} k}{(2 \pi)^{3}} \operatorname{Tr}\left[S^{>}(\vec{k} ; 0, t) S^{<}(\vec{k} ; t, 0) \Theta(-t)+S^{<}(\vec{k} ; 0, t) S^{>}(\vec{k} ; t, 0) \Theta(t)\right] .
$$

After some algebra at $T=0$, we find, assuming that $\mu>0$, and the number of colours $N$

$$
\begin{aligned}
& \mathcal{G}(t)=N_{f} N \int \frac{d^{3} k}{(2 \pi)^{3}}\left(\left[(1-\Theta(\mu-k)) e^{2 i k t}+\Theta(\mu-k) e^{-2 i k t}\right] \Theta(-t)\right. \\
&\left.+\left[\Theta(\mu-k) e^{2 i k t}+(1-\Theta(\mu-k)) e^{-2 i k t}\right] \Theta(t)\right) .
\end{aligned}
$$

The frequency space propagator,

$$
\tilde{\mathcal{G}}(\omega)=i \int_{-\infty}^{\infty} d t e^{i \omega t} \mathcal{G}(t)
$$

is therefore,

$$
\begin{aligned}
& \tilde{\mathcal{G}}(\omega)=N_{f} N \int \frac{d^{3} k}{(2 \pi)^{3}}\left[\frac{1}{\omega+2 k-i \epsilon}-\frac{1}{\omega-2 k+i \epsilon}+\right. \\
& \left.+\Theta(\mu-k)\left(\frac{1}{\omega-2 k-i \epsilon}-\frac{1}{\omega+2 k-i \epsilon}+\frac{1}{\omega-2 k+i \epsilon}-\frac{1}{\omega+2 k+i \epsilon}\right)\right] .
\end{aligned}
$$

Rewriting the energy denominators using $1 /(x-i \epsilon)=\mathcal{P}(1 / x)+i \pi \delta(x)$, we get

$$
\begin{aligned}
\tilde{\mathcal{G}}(\omega)=N_{f} N \int \frac{d^{3} k}{(2 \pi)^{3}} \quad & {\left[\left(\mathcal{P} \frac{1}{\omega+2 k}-\mathcal{P} \frac{1}{\omega-2 k}\right)(1-2 \Theta(\mu-k))\right.} \\
+ & \left.\frac{i \pi}{2} \delta\left(k+\frac{\omega}{2}\right)+\frac{i \pi}{2} \delta\left(k-\frac{\omega}{2}\right)\right] .
\end{aligned}
$$

Recalling that $k=|\vec{k}|$, the spatial momentum integrals can be performed so that,

$$
\tilde{\mathcal{G}}(\omega)=-\frac{N_{f} N}{16 \pi^{2}}\left[\omega^{2} \ln \omega^{2}+\omega^{2} \ln \left(\frac{\omega^{2}-4 \mu^{2}}{\omega^{2}}\right)^{2}\right]
$$


Here we have ignored additive constants and contact terms, analytic in $\omega$. The chemical potential has modified the $\mu=0$ result for the Green's function of $\mathcal{O}$, introducing branch points at $\omega= \pm 2 \mu$.

\section{Retarded propagator:}

From the expression for the time ordered propagator, we see that the contributions of on-shell intermediate states, even in the presence of the chemical potential, only yield singularities on the real $\omega$ axis. Therefore the different propagators time-ordered and retarded, differ only in the choice of $i \epsilon$ prescriptions for these singularities. We confirm this by carrying out the explict computation of the retarded propagator, defined as

$$
\tilde{\mathcal{G}}_{R}(\omega)=i \int_{0}^{\infty} d t e^{i \omega t} \Theta(t) \int d^{3} x\langle[\mathcal{O}(\vec{x}, t), \mathcal{O}(0)]\rangle .
$$

We find that

$$
\tilde{\mathcal{G}}_{R}(\omega)=N_{f} N \int \frac{d^{3} k}{(2 \pi)^{3}}\left[\frac{1}{\omega+2 k+i \epsilon}-\frac{1}{\omega-2 k+i \epsilon}\right](1-2 \Theta(\mu-k)) .
$$

The real part of this expression (for real $\omega$ ) is exactly the same as the time-ordered propagator. The main difference lies in the imaginary part which is determined by the $i \epsilon$ prescriptions. Up to additive constants, the retarded propagator at finite density in flat space is

$$
\tilde{\mathcal{G}}_{R}(\omega)=-\frac{N_{f} N}{16 \pi^{2}}\left[\omega^{2} \ln \omega^{2}+\omega^{2} \ln \left(\frac{4 \mu^{2}-\omega^{2}}{\omega^{2}}\right)^{2}+2 i \pi \omega^{2} \operatorname{sgn}(\omega) \Theta\left(\omega^{2}-4 \mu^{2}\right)(\mathrm{B} .16)\right.
$$

We can also deduce the form of the two-point function of $\mathcal{O}$ on $S^{3}$ from the formula (B.12) by replacing the integrals by a discrete sum over the energy levels on $S^{3}$ :

$$
\tilde{\mathcal{G}}(\omega)=\frac{N_{f} N}{2 \pi^{2} R^{3}} \sum_{\ell=1}^{\infty} d_{\ell}\left(\frac{1}{\omega+2 \varepsilon_{\ell}}-\frac{1}{\omega-2 \varepsilon_{\ell}}-2 \Theta\left(\mu-\varepsilon_{\ell}\right)\left[\frac{1}{\omega+2 \varepsilon_{\ell}}-\frac{1}{\omega-2 \varepsilon_{\ell}}\right]\right)
$$

where for $S^{3}$ fermion harmonics

$$
\varepsilon_{\ell}=\left(\ell+\frac{1}{2}\right) R^{-1}, \quad d_{\ell}=2 \ell(\ell+1)
$$

\section{Appendix C: D7-brane embedding ansatz}

In this appendix, we see why the embeddings with $g(z) \neq 0$ are not suitable for the theory with massless hypermultiplets. With the general ansatz

$$
\phi(z, t)=2 \mu t+g(z)
$$


the DBI action for the probe D7-brane becomes

$$
\begin{aligned}
S_{\mathrm{D} 7}= & 2 \pi^{2} \mathcal{N}_{\mathrm{D} 7} \int d t d z \cos ^{3} \theta \frac{\left(1-\frac{z^{2}}{4}\right)^{3}}{z^{5}} \times \\
& \sqrt{\left(1+z^{2} \theta^{\prime}(z)^{2}\right)\left(\left(1+\frac{z^{2}}{4}\right)^{2}-4 \mu^{2} z^{2} \sin ^{2} \theta\right)+g^{\prime}(z)^{2} \sin ^{2} \theta z^{2}\left(1+\frac{z^{2}}{4}\right)^{2}} .
\end{aligned}
$$

As usual, since the action does not depend on $g(z)$, we have

$$
\frac{\partial \mathcal{L}}{\partial g^{\prime}}=2 \pi^{2} \mathcal{N}_{\mathrm{D} 7} c=\text { constant }
$$

From this condition we find,

$$
g^{\prime}(z)=c \sqrt{\frac{\left(1+z^{2} \theta^{\prime}(z)^{2}\right)\left(\left(1+\frac{z^{2}}{4}\right)^{2}-4 \mu^{2} z^{2} \sin ^{2} \theta\right)}{\cos ^{6} \theta \sin ^{2} \theta\left(1+\frac{z^{2}}{4}\right)^{2}\left(1-\frac{z^{2}}{4}\right)^{6} z^{-8}-c^{2}} \frac{1}{\sin \theta\left(1+\frac{z^{2}}{4}\right) z}}
$$

The equation of motion for $\theta(z)$ is best obtained through the Legendre transformed action

$$
S_{\mathrm{LT}}=S_{\mathrm{D} 7}-2 \pi^{2} \mathcal{N}_{\mathrm{D} 7} \int d t d z g^{\prime}(z) c
$$

which simplifies to

$$
\begin{aligned}
& S_{\mathrm{LT}}=2 \pi^{2} \mathcal{N}_{\mathrm{D} 7} \int d t d z \times \frac{1}{z \sin \theta\left(1+\frac{z^{2}}{4}\right)} \\
& \sqrt{\left(1+z^{2} \theta^{\prime}(z)^{2}\right)\left(\left(1+\frac{z^{2}}{4}\right)^{2}-4 \mu^{2} z^{2} \sin ^{2} \theta\right)\left(\cos ^{6} \theta \sin ^{2} \theta\left(1+\frac{z^{2}}{4}\right)^{2}\left(1-\frac{z^{2}}{4}\right)^{6} z^{-8}-c^{2}\right)}
\end{aligned}
$$

From the equation of motion for $\theta(z)$, we find that the asymptotic behaviour of the slipping angle near the boundary is

$$
\theta(z)=\theta_{(0)} z+\theta_{(2)} z^{3}+\frac{1}{2} \theta_{(0)}\left(1-4 \mu^{2}\right) z^{3} \ln z+\ldots
$$

so that the constant of the motion $c$ does not appear in the mass or the condensate of the corresponding field theory operator. However, it does make an appearance in $g(z)$ and therefore in $\phi(z, t)$, which then has the asymptotic form,

$$
\phi(z, t) \rightarrow 2 \mu t+\frac{1}{2} \frac{c}{\theta_{(0)}^{2}} z+\ldots
$$

For massless fields this implies a putative divergence in $\phi$, near the boundary. This suggests that non-zero values of $c$ are potentially problematic for configurations with massless hypermultiplets and so $c$ must be set to zero for this case. (See [14] for a detailed explanation of related issues.) 


\section{References}

[1] J. M. Maldacena, "The large $N$ limit of superconformal field theories and supergravity," Adv. Theor. Math. Phys. 2, 231 (1998) [Int. J. Theor. Phys. 38, 1113 (1999)] [hep-th/9711200].

[2] O. Aharony, S. S. Gubser, J. M. Maldacena, H. Ooguri and Y. Oz, "Large N field theories, string theory and gravity," Phys. Rept. 323, 183 (2000) [hep-th/9905111].

[3] E. Witten, "Anti-de Sitter space, thermal phase transition, and confinement in gauge theories," Adv. Theor. Math. Phys. 2, 505 (1998) [hep-th/9803131];

E. Witten, "Anti-de Sitter space and holography," Adv. Theor. Math. Phys. 2, 253 (1998) [hep-th/9802150].

[4] S. S. Gubser, “Thermodynamics of spinning D3-branes," Nucl. Phys. B 551, 667 (1999) [hep-th/9810225];

A. Chamblin, R. Emparan, C. V. Johnson and R. C. Myers, "Charged AdS black holes and catastrophic holography," Phys. Rev. D 60, 064018 (1999)

[hep-th/9902170];

M. Cvetic and S. S. Gubser, "Phases of R-charged black holes, spinning branes and strongly coupled gauge theories," JHEP 9904, 024 (1999) [hep-th/9902195].

[5] A. Karch and L. Randall, "Open and closed string interpretation of SUSY CFT's on branes with boundaries," JHEP 0106, 063 (2001) [hep-th/0105132];

A. Karch and E. Katz, "Adding flavor to AdS/CFT," JHEP 0206, 043 (2002) [hep-th/0205236];

J. Babington, J. Erdmenger, N. J. Evans, Z. Guralnik and I. Kirsch, "Chiral symmetry breaking and pions in non-supersymmetric gauge / gravity duals," Phys. Rev. D 69, 066007 (2004) [hep-th/0306018];

I. Kirsch, "Generalizations of the AdS/CFT correspondence," Fortsch. Phys. 52, 727 (2004) [hep-th/0406274].

[6] K. Ghoroku, T. Sakaguchi, N. Uekusa and M. Yahiro, "Flavor quark at high temperature from a holographic model," Phys. Rev. D 71, 106002 (2005)

[hep-th/0502088];

D. Mateos, R. C. Myers and R. M. Thomson, "Holographic phase transitions with fundamental matter," Phys. Rev. Lett. 97, 091601 (2006) [hep-th/0605046];

T. Albash, V. G. Filev, C. V. Johnson and A. Kundu, "A topology-changing phase transition and the dynamics of flavour," Phys. Rev. D 77, 066004 (2008) [hep-th/0605088].

[7] S. Kobayashi, D. Mateos, S. Matsuura, R. C. Myers and R. M. Thomson, "Holographic phase transitions at finite baryon density," JHEP 0702, 016 (2007) [hep-th/0611099];

D. Mateos, R. C. Myers and R. M. Thomson, "Thermodynamics of the brane," JHEP 0705, 067 (2007) [hep-th/0701132]; 
K. Ghoroku, M. Ishihara and A. Nakamura, "D3/D7 holographic Gauge theory and Chemical potential," Phys. Rev. D 76, 124006 (2007) [0708.3706 [hep-th]];

S. Nakamura, Y. Seo, S. J. Sin and K. P. Yogendran, "Baryon-charge Chemical Potential in AdS/CFT," Prog. Theor. Phys. 120, 51 (2008) [0708.2818 [hep-th]]; A. Karch and A. O'Bannon, "Holographic Thermodynamics at Finite Baryon Density: Some Exact Results," JHEP 0711, 074 (2007) [0709.0570 [hep-th]]. D. Mateos, S. Matsuura, R. C. Myers and R. M. Thomson, "Holographic phase transitions at finite chemical potential," JHEP 0711, 085 (2007) [0709.1225 [hep-th]].

[8] T. Sakai and S. Sugimoto, "Low energy hadron physics in holographic QCD," Prog. Theor. Phys. 113, 843 (2005) [hep-th/0412141].

[9] O. Aharony, J. Sonnenschein and S. Yankielowicz, "A holographic model of deconfinement and chiral symmetry restoration," Annals Phys. 322, 1420 (2007) [arXiv:hep-th/0604161].

[10] N. Horigome and Y. Tanii, "Holographic chiral phase transition with chemical potential," JHEP 0701, 072 (2007) [arXiv:hep-th/0608198].

[11] S. A. Hartnoll, C. P. Herzog and G. T. Horowitz, "Building a Holographic Superconductor," Phys. Rev. Lett. 101, 031601 (2008) [arXiv:0803.3295 [hep-th]]. S. A. Hartnoll, "Lectures on holographic methods for condensed matter physics," Class. Quant. Grav. 26, 224002 (2009) [arXiv:0903.3246 [hep-th]].

[12] S. Hands, T. J. Hollowood and J. C. Myers, "QCD with Chemical Potential in a Small Hyperspherical Box,” JHEP 1007, 086 (2010) [arXiv:1003.5813 [hep-th]].

[13] S. Hands, T. J. Hollowood and J. C. Myers, "Numerical Study of the Two Color Attoworld," JHEP 1012, 057 (2010) [arXiv:1010.0790 [hep-lat]].

[14] A. O'Bannon, "Toward a Holographic Model of Superconducting Fermions," JHEP 0901, 074 (2009) [arXiv:0811.0198 [hep-th]].

[15] M. Ammon, J. Erdmenger, M. Kaminski and P. Kerner, "Superconductivity from gauge/gravity duality with flavor," Phys. Lett. B 680, 516 (2009) [arXiv:0810.2316 [hep-th]].

[16] M. Ammon, J. Erdmenger, M. Kaminski and P. Kerner, "Flavor Superconductivity from Gauge/Gravity Duality,” JHEP 0910, 067 (2009) [arXiv:0903.1864 [hep-th]].

[17] M. Ammon, J. Erdmenger, M. Kaminski and A. O'Bannon, "Fermionic Operator Mixing in Holographic p-wave Superfluids," JHEP 1005, 053 (2010) [arXiv:1003.1134 [hep-th]].

[18] R. C. Myers and M. C. Wapler, "Transport Properties of Holographic Defects," JHEP 0812, 115 (2008) [arXiv:0811.0480 [hep-th]].

[19] P. Basu, J. He, A. Mukherjee and H. H. Shieh, "Superconductivity from D3/D\%: Holographic Pion Superfluid,” JHEP 0911, 070 (2009) [arXiv:0810.3970 [hep-th]]. 
[20] N. Evans and E. Threlfall, "Chemical Potential in the Gravity Dual of a $2+1$ Dimensional System," Phys. Rev. D 79, 066008 (2009) [arXiv:0812.3273 [hep-th]]; N. Evans and E. Threlfall, "R-Charge Chemical Potential in the M2-M5 System," arXiv:0807.3679 [hep-th].

[21] O. DeWolfe, D. Z. Freedman and H. Ooguri, "Holography and defect conformal field theories," Phys. Rev. D 66, 025009 (2002) [arXiv:hep-th/0111135].

[22] N. R. Constable, J. Erdmenger, Z. Guralnik and I. Kirsch, "Intersecting D3-branes and holography," Phys. Rev. D 68, 106007 (2003) [arXiv:hep-th/0211222].

[23] G. T. Horowitz, "Introduction to Holographic Superconductors," arXiv:1002.1722 [hep-th].

[24] B. Sundborg, "The Hagedorn Transition, Deconfinement and N=4 SYM Theory," Nucl. Phys. B 573, 349 (2000) [hep-th/9908001].

[25] O. Aharony, J. Marsano, S. Minwalla, K. Papadodimas and M. Van Raamsdonk, "The Hagedorn / deconfinement phase transition in weakly coupled large $N$ gauge theories," Adv. Theor. Math. Phys. 8, 603 (2004) [hep-th/0310285].

[26] D. Yamada and L. G. Yaffe, "Phase diagram of $N=4$ super-Yang-Mills theory with R-symmetry chemical potentials," JHEP 0609, 027 (2006) [hep-th/0602074].

[27] D. Yamada, "Metastability of R-charged black holes," Class. Quant. Grav. 24, 3347 (2007) [hep-th/0701254].

[28] T. J. Hollowood, S. P. Kumar, A. Naqvi and P. Wild, "N=4 SYM on $S^{3}$ with Near Critical Chemical Potentials," JHEP 0808, 046 (2008) [0803.2822 [hep-th]].

[29] S. R. Das, T. Nishioka and T. Takayanagi, "Probe Branes, Time-dependent Couplings and Thermalization in AdS/CFT," JHEP 1007, 071 (2010) [arXiv:1005.3348 [hep-th]].

[30] T. Hollowood, S. P. Kumar and A. Naqvi, "Instabilities of the small black hole: A view from $N=4 S Y M$," JHEP 0701, 001 (2007) [hep-th/0607111].

[31] D. J. Gross and E. Witten, "Possible Third Order Phase Transition In The Large N Lattice Gauge Theory," Phys. Rev. D 21, 446 (1980).

[32] C. Fefferman and C. Robin Graham, 'Conformal Invariants,' in Elie Cartan et les Mathematiques d'aurjourd'hui (Asterique, 1985) 95.

[33] A. Karch and A. O'Bannon, "Metallic AdS/CFT," JHEP 0709, 024 (2007) [arXiv:0705.3870 [hep-th]].

[34] A. Karch, A. O'Bannon and K. Skenderis, "Holographic renormalization of probe D-branes in AdS/CFT," JHEP 0604, 015 (2006) [hep-th/0512125]. 
[35] A. O'Bannon, "Holographic Thermodynamics and Transport of Flavor Fields," 0808.1115 [hep-th].

[36] A. Karch, A. O'Bannon and L. G. Yaffe, "Critical Exponents from AdS/CFT with Flavor," JHEP 0909, 042 (2009) [arXiv:0906.4959 [hep-th]].

[37] A. Karch and A. O'Bannon, "Chiral transition of $N=4$ super Yang-Mills with flavor on a 3-sphere," Phys. Rev. D 74, 085033 (2006) [hep-th/0605120].

[38] J. Erdmenger and V. Filev, "Mesons from global Anti-de Sitter space," JHEP 1101, 119 (2011) [arXiv:1012.0496 [hep-th]].

[39] G. Basar and G. V. Dunne, "Self-consistent crystalline condensate in chiral Gross-Neveu and Bogoliubov-de Gennes systems," Phys. Rev. Lett. 100, 200404 (2008) [arXiv:0803.1501 [hep-th]].

G. Basar and G. V. Dunne, "A Twisted Kink Crystal in the Chiral Gross-Neveu model," Phys. Rev. D 78, 065022 (2008) [arXiv:0806.2659 [hep-th]].

G. Basar, G. V. Dunne and M. Thies, "Inhomogeneous Condensates in the Thermodynamics of the Chiral NJL(2) model," Phys. Rev. D 79, 105012 (2009) [arXiv:0903.1868 [hep-th]].

[40] D. J. Gross and A. Neveu, "Dynamical Symmetry Breaking in Asymptotically Free Field Theories," Phys. Rev. D 10, 3235 (1974).

[41] R. Camporesi and A. Higuchi, "On The Eigen Functions Of The Dirac Operator On Spheres And Real Hyperbolic Spaces," J. Geom. Phys. 20, 1 (1996) [arXiv:gr-qc/9505009].

$[42]$ D. Boyanovsky and H. J. de Vega, "Non-Fermi liquid aspects of cold and dense QED and QCD: Equilibrium and non-equilibrium," Phys. Rev. D 63, 034016 (2001) [arXiv:hep-ph/0009172]. 